\title{
REDUCTION OF FILTERED K-THEORY AND A CHARACTERIZATION OF CUNTZ-KRIEGER ALGEBRAS
}

\author{
SARA E. ARKLINT, RASMUS BENTMANN, AND TAKESHI KATSURA
}

\begin{abstract}
We show that filtered K-theory is equivalent to a substantially smaller invariant for all real-rank-zero $C^{*}$-algebras with certain primitive ideal spaces - including the infinitely many so-called accordion spaces for which filtered K-theory is known to be a complete invariant. As a consequence, we give a characterization of purely infinite Cuntz-Krieger algebras whose primitive ideal space is an accordion space.
\end{abstract}

\section{INTRODUCTION}

The Cuntz and Cuntz-Krieger algebras are historically and in general of great importance for our understanding of simple and non-simple purely infinite $C^{*}$-algebras as they were not only the first constructed examples of such but are also very tangible due to the combinatorial nature of their construction [12. CuntzKrieger algebras arise from shifts of finite type and it has been shown that they are exactly the graph $C^{*}$-algebras $C^{*}(E)$ arising from finite directed graphs $E$ with no sources [5]. Using the Kirchberg-Phillips classification theorem [16, 22], the Cuntz algebras and simple Cuntz-Krieger algebras can be identified, up to isomorphism, as the unital UCT Kirchberg algebras with a specific type of K-theory [11, 25]. A similar characterization for non-simple, purely infinite Cuntz-Krieger algebras and, more generally, of unital graph $C^{*}$-algebras of this type is desirable.

A Kirchberg $X$-algebra is a purely infinite, nuclear, separable $C^{*}$-algebra with primitive ideal space homeomorphic to $X$ (in a specified way). When $X$ is a so-called accordion space, see Definition 2.1, the invariant filtered K-theory FK is a strongly complete invariant for stable Kirchberg $X$-algebras with simple subquotients in the bootstrap class [8, 17, 19]. In particular, filtered K-theory is complete for purely infinite graph $C^{*}$-algebras with primitive ideal space of accordion type, and the main goal of this paper is to use this to achieve a characterization in the sense of the previous paragraph of such purely infinite Cuntz-Krieger algebras and graph $C^{*}$-algebras. Since a Cuntz-Krieger algebra is purely infinite if and only if it has real rank zero (and more generally, a purely infinite graph $C^{*}$-algebra always has real rank zero [15]), we will specifically investigate filtered K-theory for $C^{*}$-algebras of real rank zero.

In the companion paper [3, we determine the range of reduced filtered K-theory with respect to purely infinite Cuntz-Krieger algebras and graph $C^{*}$-algebras.

2010 Mathematics Subject Classification. 46L35, 46L80, (46L55).

Key words and phrases. $C^{*}$-algebras, graph $C^{*}$-algebras, classification, filtered K-theory, real rank zero.

This research was supported by the Danish National Research Foundation through the Centre for Symmetry and Deformation (DNRF92). The third-named author was partially supported by the Japan Society for the Promotion of Science. 
This invariant was originally defined by Gunnar Restorff [23], who used it to give an "internal" classification of purely infinite Cuntz-Krieger algebras, inspired by work of Mikael Rørdam [26] and work of Mike Boyle and Danrun Huang on dynamical systems [9. In the present note, we show that under some assumptions on the primitive ideal space - which are satisfied for accordion spaces - the invariants filtered K-theory and reduced filtered K-theory are in a certain sense equivalent when restricted to purely infinite graph $C^{*}$-algebras.

To be more precise, we show that isomorphisms on the reduced filtered K-theory of purely infinite graph $C^{*}$-algebras over so-called EBP spaces lift to isomorphisms on concrete filtered K-theory - this invariant may be considered as a more explicit model of filtered K-theory: the two are known to coincide for many spaces but not in general (compare Remark 5.16). Along the way, we introduce filtered K-theory restricted to the canonical base, denoted $\mathrm{FK}_{\mathcal{B}}$, and show that, for real-rank-zero $C^{*}$-algebras over an EBP space, isomorphisms on $\mathrm{FK}_{\mathcal{B}}$ lift to isomorphisms on concrete filtered K-theory.

For accordion spaces, our results furnish one-to-one correspondences, induced by the different variants of filtered $\mathrm{K}$-theory, between purely infinite graph $C^{*}$-algebras respectively unital purely infinite graph $C^{*}$-algebras or purely infinite Cuntz-Krieger algebras on the one hand, and certain types of modules in the respective target categories on the other hand. In particular, we obtain the desired characterization of purely infinite Cuntz-Krieger algebras with accordion spaces as primitive ideal spaces:

Theorem 1.1. Let $A$ be a $C^{*}$-algebra whose primitive ideal space is an accordion space. Then $A$ is a purely infinite Cuntz-Krieger algebra if and only if A satisfies the following:

- A is unital, purely infinite, nuclear, separable, and of real rank zero,

- for all ideals $I$ and $J$ of $A$ with $I \subseteq J$ and $J / I$ simple, the quotient $J / I$ belongs to the bootstrap class, the group $\mathrm{K}_{*}(J / I)$ is finitely generated, the group $\mathrm{K}_{1}(J / I)$ is free and $\operatorname{rank} \mathrm{K}_{1}(J / I)=\operatorname{rank} \mathrm{K}_{0}(J / I)$.

In the terms introduced by the first named author in [2], our Theorem 1.1] states that there is no phantom Cuntz-Krieger algebra whose primitive ideal space is an accordion space. It is an open question whether this holds for all finite primitive ideal spaces.

1.1. Historical account. By a seminal result of Eberhard Kirchberg, $\mathrm{KK}(X)$ equivalences between stable Kirchberg $X$-algebras, that is, stable, tight, $\mathcal{O}_{\infty}$-absorbing, nuclear, separable $C^{*}$-algebras over a space $X$, lift to $X$-equivariant ${ }^{*}$-isomorphisms. In [19, Ralf Meyer and Ryszard Nest established a Universal Coefficient Theorem computing the equivariant bivariant theory $\mathrm{KK}(X)$ from filtered $\mathrm{K}$-theory under the assumption that the topology of $X$ is finite and totally ordered. As a result, for such spaces $X$, isomorphisms on filtered K-theory between stable Kirchberg $X$-algebras with simple subquotients in the bootstrap class lift to $X$-equivariant ${ }^{*}$-isomorphisms. This result was generalized in [8] by the second-named author and Manuel Köhler to the case of so-called accordion spaces. Building on these results, Søren Eilers, Gunnar Restorff, and Efren Ruiz classified in [13] certain classes of real-rank-zero (not necessarily purely infinite) graph $C^{*}$-algebras using ordered filtered K-theory. 
On the other hand, Meyer-Nest and the second-named author have constructed counterexamples to the analogous classification statement over all six four-point non-accordion connected $T_{0}$-spaces. More precisely, for each of these spaces $X$, they exhibit two non-KK $(X)$-equivalent Kirchberg $X$-algebras with simple subquotients in the bootstrap class whose filtered K-theory is isomorphic (see [6, 19]).

Despite this obstruction, it had previously been shown by Gunnar Restorff in [23] that filtered K-theory - in fact reduced filtered K-theory - is a complete invariant for purely infinite Cuntz-Krieger algebras. Any finite $T_{0}$-space, in particular the six problematic four-point spaces mentioned above, can be realized as the primitive ideal space of a purely infinite Cuntz-Krieger algebra. Unfortunately, Restorff's result only gives an internal classification of Cuntz-Krieger algebras and admits no conclusion concerning when a given Cuntz-Krieger algebra is stably isomorphic to a given purely infinite, nuclear, separable $C^{*}$-algebra with the same ideal structure and filtered K-theory.

In [4, Gunnar Restorff, Efren Ruiz, and the first-named author noted that, for five of the six problematic four-point spaces, the constructed counterexamples to classification do not have real rank zero. They went on to show that for four of these spaces $X$, filtered K-theory is in fact a complete invariant for Kirchberg $X$-algebras of real rank zero with simple subquotients in the bootstrap class. The four-point non-accordion space for which the constructed counterexample does have real rank zero will be denoted by $\mathcal{D}$.

It is a general property of Cuntz-Krieger algebras that the $\mathrm{K}_{1}$-group of every subquotient is free. The same is true, more generally, for graph $C^{*}$-algebras. We observe that, for real-rank-zero $C^{*}$-algebras over $\mathcal{D}$ satisfying this K-theoretic condition, isomorphisms on the reduced filtered $\mathrm{K}$-theory lift to $\mathrm{KK}(\mathcal{D})$-equivalences (see Proposition 7.17). There are therefore no known counterexamples to classification by filtered K-theory of Kirchberg $X$-algebras with simple subquotients in the bootstrap class that have the K-theory of a real-rank-zero graph $C^{*}$-algebra.

1.2. Organization of the paper. After fixing some basic conventions and definitions in Section 2, we introduce filtered K-theory FK and concrete filtered K-theory $\mathrm{FK}_{\mathcal{S}}$ in Section 3 . Section 4 contains some basic definitions and facts concerning sheaves and cosheaves.

In Section 5, filtered K-theory restricted to the canonical base $\mathrm{FK}_{\mathcal{B}}$ is defined for spaces with the unique path property. We introduce EBP spaces and show that the concrete filtered $\mathrm{K}$-theory $\mathrm{FK}_{\mathcal{S T}}(A)$ of a real-rank-zero $C^{*}$-algebra $A$ over an EBP space is completely determined by the filtered K-theory restricted to the canonical base $\operatorname{FK}_{\mathcal{B}}(A)$, see Corollary 5.19

In Section [6, reduced filtered K-theory $\mathrm{FK}_{\mathcal{R}}$ is defined, and it is shown in Section 7 that the concrete filtered $\mathrm{K}$-theory $\mathrm{FK}_{\mathcal{S T}}(A)$ of a real-rank-zero $C^{*}$-algebra $A$ over an EBP space satisfying that all subquotients have free $\mathrm{K}_{1}$-groups can be recovered from the reduced filtered K-theory $\operatorname{FK}_{\mathcal{R}}(A)$, see Corollary 7.15, This is of particular interest because of the range results from [3] for (unital) reduced filtered K-theory on (unital) purely infinite graph $C^{*}$-algebras, see Theorem 6.12 (and 8.10). In order to proceed from reduced to concrete filtered K-theory in Section 7 , an "intermediate" invariant is introducted, which serves only technical purposes.

In Sections 8 and 9 unital filtered K-theory and ordered filtered K-theory are treated. The most complete results in our framework are possible for $C^{*}$-algebras with primitive ideal spaces of accordion type; these are summarized in Section 10 . 
1.3. Acknowledgements. Most of this work was done while the third-named author stayed at the University of Copenhagen. He would like to thank the people in Copenhagen for their hospitality. The authors are grateful to Søren Eilers for his encouragement and valuable comments. We thank Mikael Rørdam for helpful comments. The second-named author thanks Ralf Meyer for the supervision of [ 6 ] which has influenced parts of this work.

\section{NotATION}

In this article, matrices act from the right and the composite of maps $A \stackrel{f}{\rightarrow} B \stackrel{g}{\rightarrow}$ $C$ is denoted by $f g$. The category of abelian groups is denoted by $\mathfrak{A b}$, the category of $\mathbb{Z} / 2$-graded abelian groups by $\mathfrak{A} \mathfrak{b}^{\mathbb{Z} / 2}$.

Let $X$ be a finite $T_{0}$-space. For a subset $Y$ of $X$, we let $\bar{Y}$ denote the closure of $Y$ in $X$, and let $\bar{\partial} Y$ denote the boundary $\bar{Y} \backslash Y$ of $Y$. Since $X$ is a finite space, there exists a smallest open subset $\widetilde{Y}$ of $X$ containing $Y$. We let $\widetilde{\partial} Y$ denote the set $\tilde{Y} \backslash Y$. For $x, y \in X$ we write $x \leq y$ when $\overline{\{x\}} \subseteq \overline{\{y\}}$, and $x<y$ when $x \leq y$ and $x \neq y$. We write $y \rightarrow x$ when $x<y$ and no $z \in X$ satisfies $x<z<y$. A path from $y$ to $x$ is a sequence $\left(z_{k}\right)_{k=1}^{n}$ such that $z_{k+1} \rightarrow z_{k}$ for $k=1, \ldots, n-1$ and $z_{1}=x$, $z_{n}=y$. We let $\operatorname{Path}(y, x)$ denote the set of paths from $y$ to $x$.

Definition 2.1. An accordion space is a $T_{0}$-space $X=\left\{x_{1}, \ldots, x_{n}\right\}$ such that for every $k=1,2, \ldots, n-1$ either $x_{k} \rightarrow x_{k+1}$ or $x_{k} \leftarrow x_{k+1}$ holds and such that $x_{k} \rightarrow x_{l}$ does not hold for any $k, l$ with $|k-l| \neq 1$.

For instance, if $X$ is linear, that is, if $X=\left\{x_{1}, \ldots, x_{n}\right\}$ with $x_{n} \rightarrow \cdots \rightarrow x_{2} \rightarrow x_{1}$, then $X$ is an accordion space.

\section{Filtered K-THEORY}

In this section filtered K-theory and concrete filtered K-theory are defined. Some properties of objects in their target categories are introduced.

A $C^{*}$-algebra $A$ over $X$ is (equivalently given by) a $C^{*}$-algebra $A$ equipped with an infima- and suprema-preserving map $\mathbb{O}(X) \rightarrow \mathbb{}(A), U \mapsto A(U)$ mapping open subsets in $X$ to (closed, two-sided) ideals in $A$ (in particular it holds that $A(\emptyset)=0$ and $A(X)=A)$. The $C^{*}$-algebra $A$ is called tight over $X$ if the map is a latticeisomorphism. A *-homomorphism $\varphi: A \rightarrow B$ for $C^{*}$-algebras $A$ and $B$ over $X$ is called $X$-equivariant if $\varphi(A(U)) \subseteq B(U)$ for all $U \in \mathbb{O}(X)$. Let $\mathbb{L} \mathbb{C}(X)$ denote the set of locally closed subsets of $X$, that is, subsets of the form $U \backslash V$ with $U$ and $V$ open subsets of $X$ satisfying $V \subseteq U$. For $Y \in \mathbb{L} \mathbb{C}(X)$, and $U, V \in \mathbb{O}(X)$ satisfying that $Y=U \backslash V$ and $U \supseteq V$, we define $A(Y)$ as the subquotient $A(Y)=A(U) / A(V)$, which up to natural isomorphism is independent of the choice of $U$ and $V$ (see [20, Lemma 2.15]).

Definition 3.1. A tight, $\mathcal{O}_{\infty}$-absorbing, nuclear, separable $C^{*}$-algebra over $X$ is called a Kirchberg $X$-algebra.

Let $\mathfrak{K} \mathfrak{K}(X)$ be the additive category whose objects are separable $C^{*}$-algebras over $X$ and whose set of morphisms from $A$ to $B$ is the Kasparov group $\mathrm{KK}_{0}(X ; A, B)$ defined by Kirchberg (see [20, Section 3] for details). For a $C^{*}$-algebra $A$ over $X$, a $\mathbb{Z} / 2$-graded abelian group $\mathrm{FK}_{Y}^{*}(A)$ is defined as $\mathrm{K}_{*}(A(Y))$ for all $Y \in \mathbb{L} \mathbb{C}(X)$. Thus

$\mathrm{FK}_{Y}^{*}$ is an additive funtor from $\mathfrak{K} \mathfrak{K}(X)$ to the category $\mathfrak{A} \mathfrak{b}^{\mathbb{Z} / 2}$ of $\mathbb{Z} / 2$-graded abelian 
groups. Ralf Meyer and Ryszard Nest constructed in [19] $C^{*}$-algebras $R_{Y}$ over $X$ satisfying that the functors $\mathrm{FK}_{Y}^{*}$ and $\mathrm{KK}_{*}\left(X ; R_{Y},-\right)$ are naturally isomorphic.

In their definition of filtered K-theory $\mathrm{FK}^{*}$, Meyer-Nest consider the $\mathbb{Z} / 2$-graded pre-additive category $\mathcal{N} \mathcal{T}_{*}$ with objects $\mathbb{L} \mathbb{C}(X)$ and morphisms

$$
\mathrm{Nat}_{*}\left(\mathrm{FK}_{Y}^{*}, \mathrm{FK}_{Z}^{*}\right) \cong \mathrm{KK}_{*}\left(X ; R_{Z}, R_{Y}\right)
$$

between $Y$ and $Z$, where $\mathrm{Nat}_{*}\left(\mathrm{FK}_{Y}^{*}, \mathrm{FK}_{Z}^{*}\right)$ denotes the set of graded natural transformations from the functor $\mathrm{FK}_{Y}^{*}$ to the functor $\mathrm{FK}_{Z}^{*}$. The target category of $\mathrm{FK}^{*}$ is the category $\mathfrak{M o d}\left(\mathcal{N} \mathcal{T}_{*}\right)^{\mathbb{Z} / 2}$ of graded modules over $\mathcal{N} \mathcal{T}_{*}$, that is, $\mathbb{Z} / 2$-graded additive functors $\mathcal{N} \mathcal{T}_{*} \rightarrow \mathfrak{A b} \mathfrak{b}^{\mathbb{Z} / 2}$. Hence $\operatorname{FK}^{*}(A)$ consists of the groups $\operatorname{FK}_{Y}^{*}(A)$ together with the natural transformations $\mathrm{FK}_{Y}^{*}(A) \rightarrow \mathrm{FK}_{Z}^{*}(A)$.

For reasons of notation we will often find it convenient to consider instead the pre-additive category $\mathcal{N} \mathcal{T}$ with objects $\mathbb{L} \mathbb{C}(X) \times\{0,1\}$ and morphisms between $(Y, j)$ and $(Z, k)$ given by natural transformations

$$
\operatorname{Nat}\left(\mathrm{FK}_{Y}^{j} \mathrm{FK}_{Z}^{k}\right) \cong \mathrm{KK}_{0}\left(X ; \Sigma^{k} R_{Z}, \Sigma^{j} R_{Y}\right),
$$

where $\operatorname{FK}_{Y}^{j}(A)$ denotes $\mathrm{K}_{j}(A(Y))$ for $j=0,1$ and $\Sigma$ denotes suspension (with $\left.\Sigma^{0} A=A\right)$. Let $\mathfrak{M o d}(\mathcal{N T})$ denote the category of modules over $\mathcal{N} \mathcal{T}$, that is, additive functors $\mathcal{N} \mathcal{T} \rightarrow \mathfrak{A} \mathfrak{b}$.

Given a graded $\mathcal{N} \mathcal{T}_{*}$-module $M$, we define an $\mathcal{N} \mathcal{T}$-module $D(M)$ as follows: we set $D(M)(Y, i)=M(Y)_{i}$ for $(Y, i) \in \mathbb{L} \mathbb{C}(X) \times\{0,1\}$; for a morphism $f:(Y, i) \rightarrow$ $(Z, j)$ in $\mathcal{N} \mathcal{T}$, we define $D(M)(f): D(M)(Y, i) \rightarrow D(M)(Z, j)$ as the composite

$$
M(Y)_{i} \hookrightarrow M(Y)_{*} \stackrel{M(f)}{\longrightarrow} M(Z)_{*} \rightarrow M(Z)_{j} .
$$

It is straightforward to check that this yields a functor $D: \mathfrak{M o d}\left(\mathcal{N} \mathcal{T}_{*}\right)^{\mathbb{Z} / 2} \rightarrow$ $\mathfrak{M o d}(\mathcal{N} \mathcal{T})$. In fact, $D$ is an equivalence of categories - an inverse can be defined by a direct sum construction. Consequently, we define the functor FK: $\mathfrak{K} \mathfrak{K}(X) \rightarrow$ $\mathfrak{M o d}(\mathcal{N} \mathcal{T})$ as the composite $\mathrm{FK}=D \circ \mathrm{FK}^{*}$.

Definition 3.2. Let $Y \in \mathbb{L} \mathbb{C}(X), U \subseteq Y$ be open in $Y$, and set $C=Y \backslash U$. A pair $(U, C)$ obtained in this way is called a boundary pair. The natural transformations occuring in the six-term exact sequence in K-theory for the distinguished subquotient inclusion associated to $U \subseteq Y$ are denoted by $i_{U}^{Y}, r_{Y}^{C}$ and $\delta_{C}^{Y}$ :

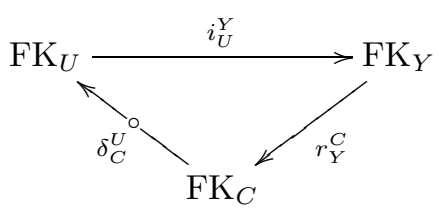

These elements $i_{U}^{Y}, r_{Y}^{C}$ and $\delta_{C}^{Y}$ correspond to the $\mathrm{KK}(X)$-classes of the ${ }^{*}$-homomorphisms $R_{Y} \rightarrow R_{U}, R_{C} \hookrightarrow R_{Y}$, and the extention $R_{C} \hookrightarrow R_{Y} \rightarrow R_{U}$, see [19]. These elements of $\mathcal{N} \mathcal{T}_{*}$ satisfy the following relations.

Proposition 3.3. In the category $\mathcal{N} \mathcal{T}_{*}$, the following relations hold.

(1) For every $Y \in \mathbb{L} \mathbb{C}(X)$,

$$
i_{Y}^{Y}=r_{Y}^{Y}=\mathrm{id}_{Y}
$$

(2) If $Y, Z \in \mathbb{L} \mathbb{C}(X)$ are topologically disjoint, then $Y \cup Z \in \mathbb{L} \mathbb{C}(X)$ and

$$
r_{Y \cup Z}^{Y} i_{Y}^{Y \cup Z}+r_{Y \cup Z}^{Z} i_{Z}^{Y \cup Z}=\operatorname{id}_{Y \cup Z} .
$$


(3) For $Y \in \mathbb{L} \mathbb{C}(X)$ and open subsets $U \subseteq V \subseteq Y$,

$$
i_{U}^{V} i_{V}^{Y}=i_{U}^{Y}
$$

(4) For $Y \in \mathbb{L} \mathbb{C}(X)$ and closed subsets $C \subseteq D \subseteq Y$,

$$
r_{Y}^{D} r_{D}^{C}=r_{Y}^{C}
$$

(5) For $Y \in \mathbb{L} \mathbb{C}(X)$, an open subset $U \subseteq Y$ and a closed subset $C \subseteq Y$,

$$
i_{U}^{Y} r_{Y}^{C}=r_{U}^{U \cap C} i_{U \cap C}^{C}
$$

(6) For a boundary pair $(U, C)$ in $X$ and an open subset $C^{\prime} \subseteq C,\left(U, C^{\prime}\right)$ is a boundary pair and we have

$$
i_{C^{\prime}}^{C} \delta_{C}^{U}=\delta_{C^{\prime}}^{U} .
$$

(7) For a boundary pair $(U, C)$ in $X$ and a closed subset $U^{\prime} \subseteq U,\left(U^{\prime}, C\right)$ is a boundary pair and we have

$$
\delta_{C}^{U} r_{U}^{U^{\prime}}=\delta_{C}^{U^{\prime}}
$$

(8) For $Y, Z, W \in \mathbb{L} \mathbb{C}(X)$ such that $Y \cup W \in \mathbb{L} \mathbb{C}(X)$ containing $Y$, $W$ as closed subsets, $Z \cup W \in \mathbb{L} \mathbb{C}(X)$ containing $Z, W$ as open subsets, and $W \subseteq Y \cup Z$, we have

$$
\delta_{Y}^{W \backslash Y} i_{W \backslash Y}^{Z}=r_{Y}^{W \backslash Z} \delta_{W \backslash Z}^{Z} .
$$

Proof. We only prove (8), because the other relations can be proved similarly and more easily (their proofs can be found in [6, Section 3.2]).

Let us take $Y, Z, W \in \mathbb{L} \mathbb{C}(X)$ as in (8), Let us also take a $C^{*}$-algebra $A$ over $X$. Since both $Y$ and $W$ are closed subsets of $Y \cup W \in \mathbb{L} \mathbb{C}(X), Y \cap W$ is closed both in $Y$ and in $W$. Therefore we have a commutative diagram with exact rows

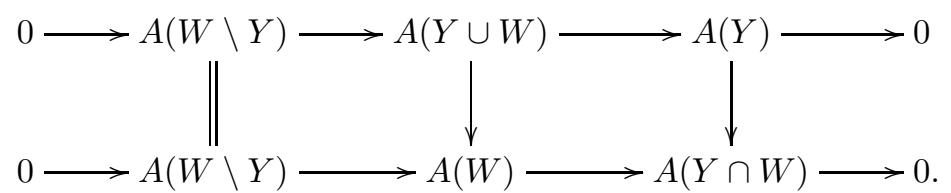

Since both $Z$ and $W$ are open subsets of $Z \cup W \in \mathbb{L} \mathbb{C}(X), Z \cap W$ is open both in $Z$ and in $W$. Therefore we have a commutative diagram with exact rows

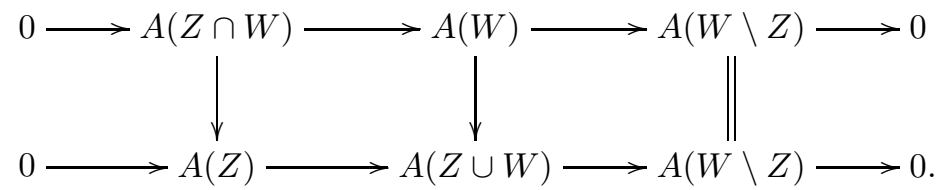

From $W \subseteq Y \cup Z$, we get $W \backslash Y \subseteq Z \cap W$ and $W \backslash Z \subseteq Y \cap W$. Since $W \backslash Y$ is open in $W$, we see that $W \backslash Y$ is open in $Z \cap W$. Similarly, $W \backslash Z$ is closed in $Y \cap W$. Hence we get a commutative diagram with exact rows

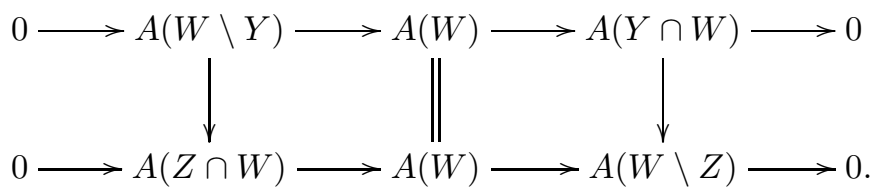


By combining these three diagrams, we obtain a commutative diagram with exact rows

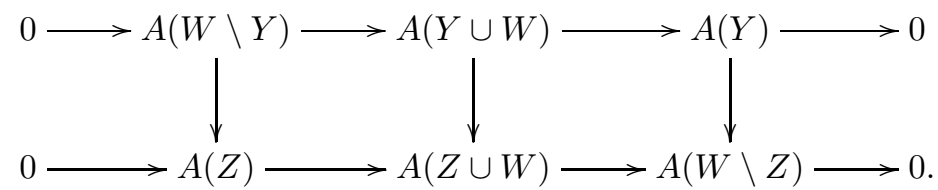

From this digram, we get a commutative diagram

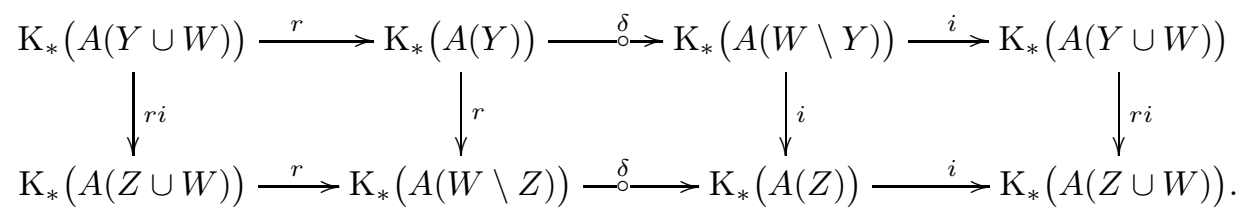

Now (8) follows from the commutativity of the middle square of this natural diagram.

Remark 3.4. From Proposition 3.3](2), we see that the empty set $\emptyset$ is a zero object in $\mathcal{N} \mathcal{T}_{*}$ (because initial objects in pre-additive categories are also terminal). From this and other relations in Proposition 3.3. we can conclude that compositions of consecutive maps in six-term sequences associated to relatively open subset inclusions vanish.

Remark 3.5. We usually denote the even and the odd component of the element $i_{U}^{Y}$ in $\mathcal{N} \mathcal{T}_{*}$ defined in Definition 3.2 simply by $i_{U}^{Y}$. Often, sub- and superscripts are suppressed when clear from context. Similar comments apply to $r$ and $\delta$.

Definition 3.6. Let $\mathcal{S} \mathcal{T}_{*}$ be the universal $\mathbb{Z} / 2$-graded pre-additive category whose set of objects is $\mathbb{L} \mathbb{C}(X)$ and whose set of morphisms are generated by elements as in Definition 3.2 with the relations as in Proposition 3.3. Let $\mathcal{S T}$ be the corresponding pre-additive category with object set $\mathbb{L} \mathbb{C}(X) \times\{0,1\}$.

By Proposition [3.3, we have a canonical additive functor $\mathcal{S T} \rightarrow \mathcal{N} \mathcal{T}$. This functor has been shown to be an isomorphism in all examples which have been investigated - including accordion spaces and all four-point spaces (see [6, 19]). However there is an example $Q$ of a finite $T_{0}$-space for which the functor $\mathcal{S T} \rightarrow \mathcal{N T}$ seems to be non-faithful (see Remark [5.16). For such spaces one would need to modify the definition of the category $\mathcal{S T}$, but we do not pursue this problem in this paper.

Let $\mathfrak{F}_{\mathcal{S T}}: \mathfrak{M}_{\mathfrak{o d}}(\mathcal{N} \mathcal{T}) \rightarrow \mathfrak{M}_{\mathfrak{N} d}(\mathcal{S T})$ be the functor induced by the canonical functor $\mathcal{S T} \rightarrow \mathcal{N} \mathcal{T}$.

Definition 3.7. We define concrete filtered K-theory $\mathrm{FK}_{\mathcal{S} \mathcal{T}}: \mathfrak{K} \mathfrak{K}(X) \rightarrow \mathfrak{M}$ od $(\mathcal{S T})$ as the composition $\mathfrak{F}_{\mathcal{S} \mathcal{T}} \circ \mathrm{FK}$.

Remark 3.8. As noted above, filtered K-theory FK and concrete filtered K-theory $\mathrm{FK}_{\mathcal{S} \mathcal{T}}$ coincide for accordion spaces and all four-point spaces. 
Definition 3.9. An $\mathcal{N} \mathcal{T}$-module $M$ is called exact if, for all $Y \in \mathbb{L} \mathbb{C}(X)$ and $U \in \mathbb{O}(Y)$, the sequence

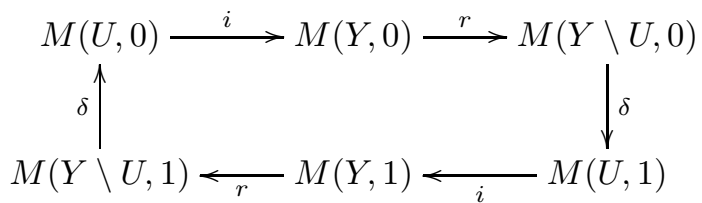

is exact. An $\mathcal{N} \mathcal{T}$-module $M$ is called real-rank-zero-like if, for all $Y \in \mathbb{L} \mathbb{C}(X)$ and $U \in \mathbb{O}(Y)$, the map $\delta: M(Y \backslash U, 0) \rightarrow M(U, 1)$ vanishes.

In the same way, we define exact $\mathcal{S} \mathcal{T}$-modules and real-rank-zero-like $\mathcal{S} \mathcal{T}$-modules.

Remark 3.10. For a $C^{*}$-algebra $A$ over $X$, the module $\operatorname{FK}(A)$ is exact. It follows from [7. Lemma 3.4] that, if $A$ is tight over $X$, then $\operatorname{FK}(A)$ is real-rank-zero-like if and only if the underlying $C^{*}$-algebra of $A$ is $\mathrm{K}_{0}$-liftable in the sense of PasnicuRørdam [21. By [18, Proposition 4], all real-rank-zero $C^{*}$-algebras are $\mathrm{K}_{0}$-liftable. By Theorem 4.2 and Example 4.8 of [21, a tight, purely infinite $C^{*}$-algebra $A$ over $X$ has real rank zero if and only if $\operatorname{FK}(A)$ is real-rank-zero-like. Analogous remarks apply with $\mathrm{FK}_{\mathcal{S} \mathcal{T}}(A)$ in place of $\mathrm{FK}(A)$.

The following theorem is the basis for the corollaries obtained in Section 10 .

Theorem 3.11 (8, 17, 19]). Let $X$ be an accordion space. The canonical functor $\mathcal{S T} \rightarrow \mathcal{N} \mathcal{T}$ is an isomorphism. Moreover, if $A$ and $B$ are stable Kirchberg $X$-algebras with all simple subquotients in the bootstrap class, then any isomorphism $\mathrm{FK}(A) \rightarrow \mathrm{FK}(B)$ lifts to an $X$-equivariant ${ }^{*}$-isomorphism $A \rightarrow B$.

\section{SHEAVES}

In this section we introduce sheaves and cosheaves and recall that it suffices to specify them on a basis for the topology.

Let $X$ be an arbitrary topological space. Let $\mathbb{B}$ be a basis for the topology on $X$. We note that the set $\mathbb{O}$ of all open subsets is the largest basis for the topology on $X$. We also note that for a finite space $X$, the collection $\{\widetilde{\{x\}} \mid x \in X\}$ is an example of a basis. The set $\mathbb{B}$ is a category whose morphisms are inclusions.

Definition 4.1. A covering of a set $U \in \mathbb{B}$ is a collection $\left\{U_{j}\right\}_{j \in J} \subseteq \mathbb{B}$ such that $U_{j} \subseteq U$ for all $j \in J$ and $\bigcup_{j \in J} U_{j}=U$. A presheaf on $\mathbb{B}$ is a contravariant functor $M: \mathbb{B} \rightarrow \mathfrak{A} \mathfrak{b}$. It is a sheaf on $\mathbb{B}$ if, for every $U \in \mathbb{B}$, every covering $\left\{U_{j}\right\}_{j \in J} \subseteq \mathbb{B}$ of $U$, and all coverings $\left\{U_{j k l}\right\}_{l \in L_{j k}} \subseteq \mathbb{B}$ of $U_{j} \cap U_{k}$, the sequence

$$
0 \longrightarrow M(U) \stackrel{\left(M\left(i_{U}^{U_{j}}\right)\right)}{\longrightarrow} \prod_{j \in J} M\left(U_{j}\right) \stackrel{\left(M\left(i_{U_{j}}^{U_{j k l}}\right)-M\left(i_{U_{k}}^{U_{j k l}}\right)\right)}{\longrightarrow} \prod_{j, k \in J} \prod_{l \in L_{j k}} M\left(U_{j k l}\right)
$$

is exact. A morphism for sheaves is a natural transformation of functors. We denote by $\mathfrak{S h}(\mathbb{B})$ the category of sheaves on $\mathbb{B}$. 
If $\mathbb{B}$ is closed under intersection (for example if $\mathbb{B}=\mathbb{O}$ ), then the definition of sheaf can be replaced with the exactness of the sequence

$$
0 \longrightarrow M(U) \stackrel{\left(M\left(i_{U}^{U_{j}}\right)\right)}{\longrightarrow} \prod_{j \in J} M\left(U_{j}\right) \stackrel{\left(M\left(i_{U_{j}}^{U_{j} \cap U_{k}}\right)-M\left(i_{U_{k}}^{U_{j} \cap U_{k}}\right)\right)}{\longrightarrow} \prod_{j, k \in J} M\left(U_{j} \cap U_{k}\right)
$$

for all $U \in \mathbb{B}$ and every covering $\left\{U_{j}\right\}_{j \in J} \subseteq \mathbb{B}$ of $U$.

Lemma 4.3. For a basis $\mathbb{B}$ for the topology on $X$, the restriction functor $\mathfrak{S h}(\mathbb{O}) \rightarrow$ $\mathfrak{S h}(\mathbb{B})$ is an equivalence of categories.

Proof. This is a well-known fact in algebraic geometry (see, for instance the encyclopedic treatment in [27, Lemma 0090]). We confine ourselves on mentioning that (4.2) provides a formula for computing $M(U)$ for an arbitrary open subset $U$.

Definition 4.4. A precosheaf on $\mathbb{B}$ is a covariant functor $M: \mathbb{B} \rightarrow \mathfrak{A} \mathfrak{b}$. It is a cosheaf on $\mathbb{B}$ if, for every $U \in \mathbb{B}$, every covering $\left\{U_{j}\right\}_{j \in J} \subseteq \mathbb{B}$ of $U$, and all coverings $\left\{U_{j k l}\right\}_{l \in L_{j k}} \subseteq \mathbb{B}$ of $U_{j} \cap U_{k}$, the sequence

$$
\bigoplus_{j, k \in J} \bigoplus_{l \in L_{j k}} M\left(U_{j k l}\right) \stackrel{\left(M\left(i_{U_{j k l}}^{U_{j}}\right)-M\left(i_{U_{j k l}}^{U_{k}}\right)\right)}{\longrightarrow} \bigoplus_{j \in J} M\left(U_{j}\right) \stackrel{\left(M\left(i_{U_{j}}^{U}\right)\right)}{\longrightarrow} M(U) \longrightarrow 0 .
$$

is exact. A morphism for cosheaves is a natural transformation of functors. We denote by $\mathfrak{C o S h}(\mathbb{B})$ the category of cosheaves on $\mathbb{B}$.

Similarly to the case of sheaves, if $\mathbb{B}$ is closed under intersection, the definition of cosheaf can be replaced with the exactness of the sequence

$$
\bigoplus_{j, k \in J} M\left(U_{j} \cap U_{k}\right) \stackrel{\left(M\left(i_{U_{j} \cap U_{k}}^{U_{j}}\right)-M\left(i_{U_{j} \cap U_{k}}^{U_{k}}\right)\right)}{\longrightarrow} \bigoplus_{j \in J} M\left(U_{j}\right) \stackrel{\left(M\left(i_{U_{j}}^{U}\right)\right)}{\longrightarrow} M(U) \longrightarrow 0 .
$$

for $U \in \mathbb{B}$ and a covering $\left\{U_{j}\right\}_{j \in J} \subseteq \mathbb{B}$ of $U$.

Lemma 4.7. The restriction functor $\mathfrak{C o S h}(\mathbb{O}) \rightarrow \mathfrak{C o S h}(\mathbb{B})$ is an equivalence of categories.

Proof. This statement is the dual of Lemma 4.3 and follows in an analogous way. Again, (4.5) can be used to compute $M(U)$ for an arbitrary open subset $U$.

With regard to the next section we remark that every finite $T_{0}$-space (more generally every Alexandrov space) comes with canonical bases for the open subsets, namely $\{\widetilde{\{x\}} \mid x \in X\}$, and for the closed subsets: $\{\overline{\{x\}} \mid x \in X\}$.

Lemma 4.8. Let $X$ be a finite $T_{0}$-space and let $S$ be a pre(co)sheaf on the basis $\mathbb{B}=\{\widetilde{\{x\}} \mid x \in X\}$. Then $S$ is a (co) sheaf.

Proof. This follows from the observation that in the basis $\mathbb{B}$ there are no non-trivial coverings, that is, if $\mathcal{U}$ is a covering of $U$, then $U \in \mathcal{U}$. 


\section{Filtered K-theory Restricted to the CANONiCAL BASE}

In this section, the functor $\mathrm{FK}_{\mathcal{B}}$ and the notions of unique path spaces and EBP spaces are introduced. The following lemma is straightforward to verify.

Lemma 5.1. For a finite $T_{0}$-space $X$ the following conditions are equivalent.

- For all $x, y \in X$, there is at most one path from $y$ to $x$.

- There are no elements $a, b, c, d$ in $X$ with $a<b<d, a<c<d$ and neither $b \leq c$ nor $c \leq b$.

- For all $x, y \in X$ with $x \rightarrow y$, we have $\overline{\{x\}} \cup \overline{\{y\}} \in \mathbb{L} \mathbb{C}(X)$.

- For every boundary pair $(U, C)$, the pair $(\widetilde{U}, \bar{C})$ is a boundary pair.

- For all $x \in X, \widetilde{\partial}\{x\}=\bigsqcup_{y \rightarrow x} \widehat{\{y\}}$.

- For all $x \in X, \bar{\partial}\{x\}=\bigsqcup_{y \leftarrow x} \overline{\{y\}}$.

Definition 5.2. A finite $T_{0}$-space $X$ is called a unique path space if it satisfies the equivalent conditions specified in Lemma 5.1 .

Let $X$ be a unique path space.

Definition 5.3. Let $\mathcal{B}$ denote the universal pre-additive category generated by objects $\bar{x}_{1}, \widetilde{x}_{0}$ for all $x \in X$ and morphisms $r_{\bar{x}_{1}}^{\bar{y}_{1}}, \delta_{\bar{y}_{1}}^{\widetilde{x}_{0}}$ and $i_{\widetilde{x}_{0}}^{\widetilde{y}_{0}}$ when $x \rightarrow y$, subject to the relations

$$
\sum_{x \rightarrow y} r_{\bar{x}_{1}}^{\bar{y}_{1}} \delta_{\bar{y}_{1}}^{\widetilde{x}_{0}}=\sum_{z \rightarrow x} \delta_{\bar{x}_{1}}^{\widetilde{z}_{0}} i_{\widetilde{z}_{0}}^{\widetilde{x}_{0}}
$$

for all $x \in X$.

Lemma 5.5. In the category $\mathcal{S} \mathcal{T}$, we have the relation

$$
\sum_{x \rightarrow y} r \frac{\overline{\{y\}}}{\{x\}} \delta \frac{\widetilde{\{x\}}}{\{y\}}=\sum_{z \rightarrow x} \delta \frac{\widetilde{\{z\}}}{\{x\}} \frac{\widetilde{\{x\}}}{\{z\}}
$$

for all $x \in X$.

Proof. Since $X$ is a unique path space, the collections $(\overline{\{y\}})_{x \rightarrow y}$ and $(\widetilde{\{z\}})_{z \rightarrow x}$ are disjoint, respectively. Hence the desired relation simplifies to

$$
r \frac{\bar{\partial}\{x\}}{\{x\}} \delta \frac{\widetilde{\{x\}}}{\partial\{x\}}=\delta \frac{\widetilde{\partial}\{x\}}{\{x\}} i_{\widetilde{\partial}\{x\}}^{\widetilde{\{x\}}}
$$

which follows from Proposition $3.3[(8)$ by setting $Y=\overline{\{x\}}, Z=\widetilde{\{x\}}$ and $W=$ $\overline{\{x\}} \cup \widetilde{\{x\}}$.

Definition 5.6. The previous lemma allows us to define an additive functor $\mathcal{B} \rightarrow$ $\mathcal{S T}$ by $\bar{x}_{1} \mapsto(\overline{\{x\}}, 1)$ and $\widetilde{x}_{0} \mapsto(\widetilde{\{x\}}, 0)$, and in the obvious way on morphisms. Let

$$
\mathfrak{F}_{\mathcal{B}}: \mathfrak{M o d}(\mathcal{S T}) \rightarrow \mathfrak{M o d}(\mathcal{B})
$$

denote the induced functor. Define filtered $\mathrm{K}$-theory restricted to the canonical base, $\mathrm{FK}_{\mathcal{B}}: \mathfrak{K} \mathfrak{K}(X) \rightarrow \mathfrak{M o d}(\mathcal{B})$, as the composition of $\mathrm{FK}_{\mathcal{S} \mathcal{T}}$ with $\mathfrak{F}_{\mathcal{B}}$. 
Remark 5.7. The invariant $\mathrm{FK}_{\mathcal{B}}$ is only defined for unique path spaces because the boundary map $\delta \frac{\{x\}}{\{y\}}$ only exists when $\overline{\{y\}} \cup \widetilde{\{x\}}$ belongs to $\mathbb{L} \mathbb{C}(X)$. We also point out that the invariant $\mathrm{FK}_{\mathcal{B}}$ can only be expected to be very useful for spaces such that the relation (5.14) holds for all boundary pairs $(U, C)$.

Definition 5.8. A $\mathcal{B}$-module $M$ is called exact if the sequence

$$
M\left(\bar{x}_{1}\right) \stackrel{\left(r_{\bar{x}_{1}}^{\bar{y}_{1}}-\delta_{\bar{x}_{1}}^{\widetilde{z}_{0}}\right.}{\longrightarrow} \bigoplus_{x \rightarrow y} M\left(\bar{y}_{1}\right) \oplus \bigoplus_{z \rightarrow x} M\left(\widetilde{z}_{0}\right) \stackrel{\left(\begin{array}{l}
\delta_{\bar{x}_{1}}^{\widetilde{x}_{0}} \\
i_{\widetilde{z}_{0}}^{x_{0}}
\end{array}\right)}{\longrightarrow} M\left(\widetilde{x}_{0}\right)
$$

is exact for all $x \in X$.

Lemma 5.10. If $M$ is an exact $\mathcal{S} \mathcal{T}$-module, then $\mathfrak{F}_{\mathcal{B}}(M)$ is an exact $\mathcal{B}$-module. In particular, if $A$ is a $C^{*}$-algebra over $X$, then the $\mathcal{B}$-module $\operatorname{FK}_{\mathcal{B}}(A)$ is exact.

Proof. Using again that the collections $(\overline{\{y\}})_{x \rightarrow y}$ and $(\overline{\{z\}})_{z \rightarrow x}$ are respectively disjoint, it suffices to prove exactness of the sequence

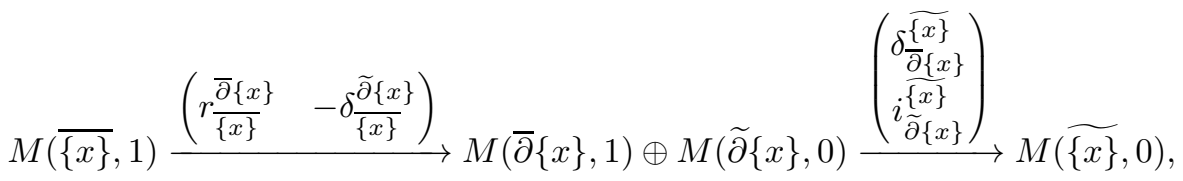

which follows from a diagram chase through the commutative diagram

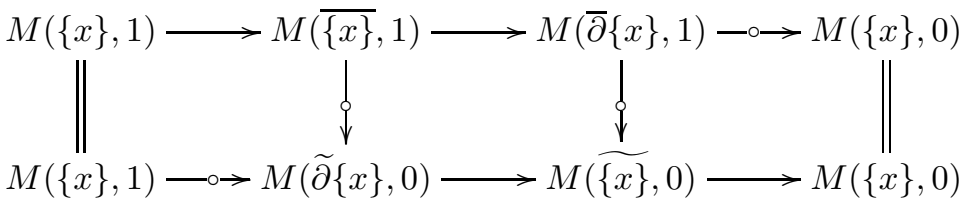

whose rows are exact.

Definition 5.11. Let $X$ be a finite $T_{0}$-space. A boundary pair $(U, C)$ in $X$ is called elementary if $U$ and $C$ are connected, $U$ is open, $C$ is closed and if, moreover, $U \subseteq \widetilde{C}$ and $C \subseteq \bar{U}$.

Definition 5.12. A unique path space $X$ is called an $E B P$ space if every elementary boundary pair $(U, C)$ in $X$ is of the form $(\widetilde{\{x\}}, \overline{\{y\}})$ for two points $x$ and $y$ in $X$ with $x \rightarrow y$.

Lemma 5.13. Let $X$ be an EBP space, and let $(U, C)$ be a boundary pair in $X$. Then the following relation holds in the category $\mathcal{S} \mathcal{T}_{*}$ :

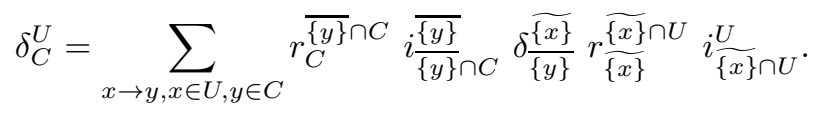

Proof. We would like to show the relation (5.14) for a boundary pair $(U, C)$ in $X$. The proof goes by the induction on the number $|U \cup C|$ of elements of $U \cup C$. If either $U$ or $C$ is empty, then both sides of (5.14) are 0 . This takes care of the case $|U \cup C|=0$. Suppose for a natural number $n$, we have shown (5.14) for all boundary pairs $(U, C)$ with $|U \cup C| \leq n$, and take a boundary pair $(U, C)$ with $|U \cup C|=n+1$, arbitrarily. We are going to show (5.14) for this pair. If either $U$ or 
$C$ is empty, again both sides of (5.14) are zero. So we may assume that both $U$ and $C$ are non-empty. Suppose $U$ is not connected, and choose two non-empty open and closed subsets $U_{1}$ and $U_{2}$ of $U$ such that $U=U_{1} \sqcup U_{2}$. Then for $i=1,2,\left(U_{i}, C\right)$ is a boundary pair with $\left|U_{i} \cup C\right| \leq n$. Thus by the assumption of the induction, both $\left(U_{1}, C\right)$ and $\left(U_{2}, C\right)$ satisfy (5.14). Hence by $(2),(7)$ and $(3)$ of Proposition 3.3 we have

$$
\begin{aligned}
& \delta_{C}^{U}=\delta_{C}^{U}\left(r_{U}^{U_{1}} i_{U_{1}}^{U}+r_{U}^{U_{2}} i_{U_{2}}^{U}\right) \\
& =\delta_{C}^{U_{1}} i_{U_{1}}^{U}+\delta_{C}^{U_{2}} i_{U_{2}}^{U} \\
& \left.=\left(\sum_{x \rightarrow y, x \in U_{1}, y \in C} r_{C}^{\overline{\{y\}} \cap C} i \frac{\overline{\{y\}}}{\{y\}} \cap C\right) \frac{\widetilde{\{x\}}}{\{y\}} r \frac{\widetilde{\{x\}} \cap U_{1}}{\{x\}} i \frac{U_{1}}{\{x\} \cap U_{1}}\right) i_{U_{1}}^{U} \\
& +\left(\sum_{x \rightarrow y, x \in U_{2}, y \in C} r_{C}^{\overline{\{y\}} \cap C} i_{\frac{\{y\}}{\{y\}} \cap C} \delta \frac{\widetilde{\{x\}}}{\{y\}} r \frac{\widetilde{\{x\}} \cap U_{2}}{\{x\}} i \frac{U_{2}}{\{x\} \cap U_{2}}\right) i_{U_{2}}^{U} \\
& \left.=\sum_{x \rightarrow y, x \in U, y \in C} r_{C}^{\overline{\{y\}} \cap C} i \frac{\overline{\{y\}}}{\{y\}} \cap C\right) \frac{\widetilde{\{x\}}}{\{y\}} r_{\{x\}}^{\widetilde{\{x\}}} \cap U \frac{U}{\{x\}} \cap U
\end{aligned}
$$

since we for $x \in U_{i}$ have $\widetilde{\{x\}} \cap U_{1}=\widetilde{\{x\}} \cap U$ because $U_{i} \subseteq U$ is open. This shows (5.14) for $(U, C)$. Thus we may now assume $U$ is connected. In a very similar way, we get (5.14) using the assumption of the induction if $C$ is not connected. Thus we may assume $C$ is connected. Next suppose we have $U \nsubseteq \widetilde{C}$. Set $U^{\prime}=U \cap \widetilde{C}$ which is a proper open subset of $U$. The pair $\left(U^{\prime}, C\right)$ is a boundary pair because $U^{\prime} \cup C=$ $(U \cup C) \cap \widetilde{C} \in \mathbb{L} \mathbb{C}(X)$. We have $\delta_{C}^{U}=\delta_{C}^{U^{\prime}} i_{U^{\prime}}^{U}$ by applying (8) of Proposition 3.3 for $Y=C, Z=U$ and $W=U^{\prime} \cup C$. Since $\left|U^{\prime} \cup C\right| \leq n$, we get by the assumption of the induction that

$$
\begin{aligned}
& \delta_{C}^{U}=\delta_{C}^{U^{\prime}} i_{U^{\prime}}^{U} \\
& \left.=\left(\sum_{x \rightarrow y, x \in U^{\prime}, y \in C} r_{C}^{\overline{\{y\}} \cap C} i \frac{\overline{\{y\}}}{\{y\}} \cap C\right) \delta \frac{\widetilde{\{x\}}}{\{y\}} r \frac{\widetilde{\{x\}}}{\{x\}} U^{\prime} i \frac{U^{\prime}}{\{x\} \cap U^{\prime}}\right) i_{U^{\prime}}^{U} \\
& \left.=\sum_{x \rightarrow y, x \in U, y \in C} r_{C}^{\overline{\{y\}} \cap C} i \frac{\overline{\{y\}}}{\{y\}} \cap C\right) \delta \overline{\frac{\{x\}}{\{y\}}} r \frac{\widetilde{\{x\}} \cap U}{\{x\}} i \frac{U}{\{x\} \cap U}
\end{aligned}
$$

since $x \rightarrow y, x \in U$ and $y \in C$ imply $x \in U^{\prime}$, and we have $\widetilde{\{x\}} \cap U=\widetilde{\{x\}} \cap U^{\prime}$. This shows (5.14) for $(U, C)$. Thus we may now assume $U \subseteq \widetilde{C}$. In a very similar way, we get (5.14) using the assumption of the induction if $C \nsubseteq \bar{U}$. Thus we may assume $C \subseteq \bar{U}$.

It remains to show (5.14) for a boundary pair $(U, C)$ such that $U$ and $C$ are connected, $U \subseteq \widetilde{C}$ and $C \subseteq \bar{U}$. To this end, we use the assumption of the lemma. Take such a pair $(U, C)$. Since $X$ is a unique path space, the pair $(\widetilde{U}, \bar{C})$ is a boundary pair by Lemma [5.1. It is not difficult to see that the pair $(\widetilde{U}, \bar{C})$ is elementary. Hence by the assumption of the lemma, there exist $x \in \widetilde{U}$ and $y \in \bar{C}$ such that $\widetilde{U}=\widetilde{\{x\}}, \bar{C}=\overline{\{y\}}$ and $x \rightarrow y$. By $[(6)$ and $[(7)$ of Proposition 3.3, we get

$$
\delta_{C}^{U}=i_{C}^{\bar{C}} \delta \frac{\widetilde{U}}{C} r_{\widetilde{U}}^{U}=i_{C}^{\overline{\{y\}}} \delta \frac{\widetilde{\{x\}}}{\{y\}} r \frac{U}{\{x\}} .
$$

It remains to prove that $(x, y)$ is the only pair satisfying $x \rightarrow y, x \in U$ and $y \in C$. First note that $\widetilde{U}=\widetilde{\{x\}}$ implies $x \in U$, and also that $\bar{C}=\overline{\{y\}}$ implies $y \in C$. Now 
take $u \in U$ and $c \in C$ with $u \rightarrow c$. Since $U \subseteq \widetilde{\{x\}}$ and $C \subseteq \overline{\{y\}}$, there exist a path from $u$ to $x$, and a path from $y$ to $c$. These two paths together with the arrow $x \rightarrow y$ give us a path from $u$ to $c$. Since $X$ is a unique path space, this path should coincide with the arrow $u \rightarrow c$. Hence we get $u=x$ and $c=y$. This finishes the proof.

Lemma 5.15. Let $X$ be a finite $T_{0}$-space. Assume that the directed graph associated to $X$ is a forrest, that is, it contains no undirected cycles. Then $X$ is an EBP space.

Proof. It is clear that, if the directed graph associated to $X$ is a forrest, then $X$ is a unique path space. Let us take an elementary boundary pair $(U, C)$. Choose a minimal element $x \in U$. Since $U \subseteq \widetilde{C}$, there is $y \in C$ with $x>y$. We can, moreover, assume that $x \rightarrow y$ because $U \cup C$ is locally closed and $x$ is minimal in $U$. Since $U$ is open and $C$ is closed, we have $\overline{\{x\}} \subseteq U$ and $\overline{\{y\}} \subseteq C$. We will show that these inclusions are equalities using the fact that $X$ is a forrest. Take $u \in U$ arbitrarily. Since $U \subseteq \widetilde{C}$, there exists an element $c \in C$ such that $u>c$. Thus we have a path from $u$ to $c$. Since both $U$ and $C$ are connected, there exist undirected paths from $u$ to $x$ and from $y$ to $c$. These two paths give us an undirected path from $u$ to $c$ through the arrow $x \rightarrow y$. This path should coincide with the directed path from $u$ to $c$ because $X$ contains no undirected cycles. Hence we get a path from $u$ to $x$. This shows $u \in \widetilde{\{x\}}$, and therefore we get $U=\widetilde{\{x\}}$. In a similar manner, we get $C=\overline{\{y\}}$.

Remark 5.16. The above lemma applies, in particular, to accordion spaces. The conclusion of Lemma 5.13 can also be verified for various unique path spaces which are not forrests - the smallest example being the so-called pseudocircle with four points. Consider, however, the sixteen-point space $Q$ defined by the directed graph

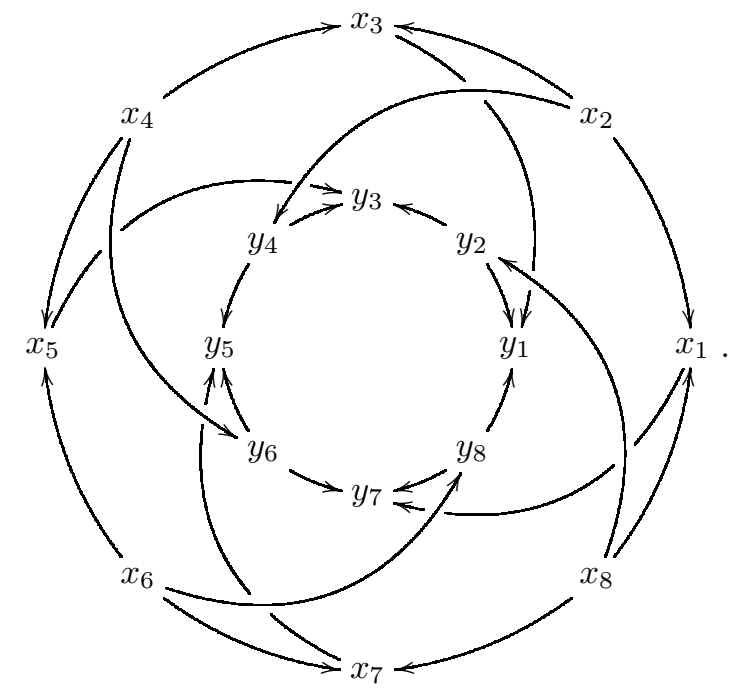

Then $Q$ is a unique path space that is not an EBP space because the subsets $U=\left\{x_{1}, x_{2}, \ldots, x_{8}\right\}$ and $C=\left\{y_{1}, y_{2}, \ldots, y_{8}\right\}$ give an elementary boundary pair $(U, C)$ that does not satisfy $U=\overline{\{x\}}$ nor $C=\overline{\{y\}}$ for any $x, y \in X$. A simple computation shows that the boundary decomposition (5.14) of $\delta_{C}^{U}$ indeed holds in the category $\mathcal{N} \mathcal{T}_{*}$. However, we believe that it does not hold in the category $\mathcal{S} \mathcal{T}_{*}$. 
The following theorem has two important consequences. Firstly, as stated in Corollary 5.19, it implies that for real-rank-zero $C^{*}$-algebras, isomorphisms on $\mathrm{FK}_{\mathcal{B}}$ lift to isomorphisms on $\mathrm{FK}_{\mathcal{S T}}$. By Theorem 3.11. $\mathrm{FK}_{\mathcal{S} \mathcal{T}}$ is strongly complete for stable Kirchberg $X$-algebras when $X$ is an accordion space. Secondly, by Lemma 5.6 of [8], if $X$ is an accordion space, any exact $\mathcal{N} \mathcal{T}$-module is of the form $\mathrm{FK}(A)$ for some Kirchberg $X$-algebra $A$, so any exact $\mathcal{B}$-module is of the form $\operatorname{FK}_{\mathcal{B}}(A)$ for some Kirchberg $X$-algebra $A$ of real rank zero. This second consequence is useful for constructing examples of Kirchberg $X$-algebras.

Theorem 5.17. Let $X$ be an EBP space. The functor

$$
\mathfrak{F}_{\mathcal{B}}: \mathfrak{M o d}(\mathcal{S T}) \rightarrow \mathfrak{M o d}(\mathcal{B})
$$

restricts to an equivalence between the category of exact real-rank-zero-like $\mathcal{S} \mathcal{T}$ modules and the category of exact $\mathcal{B}$-modules.

A proof of this theorem is given after the following remark and corollary.

Remark 5.18. The proof of Theorem 5.17 given below works in fact not only for EBP spaces but more generally for unique path spaces for which the relation (5.14) holds in the category $\mathcal{S} \mathcal{T}$ for all boundary pairs $(U, C)$, see Lemma 5.13 and Remark 5.16

Corollary 5.19. Let $A$ and $B$ be $C^{*}$-algebras of real rank zero over an EBP space $X$. Then for any homomorphism $\varphi: \mathrm{FK}_{\mathcal{B}}(A) \rightarrow \mathrm{FK}_{\mathcal{B}}(B)$, there exists a unique homomorphism $\Phi: \operatorname{FK}_{\mathcal{S T}}(A) \rightarrow \mathrm{FK}_{\mathcal{S T}}(B)$ such that $\mathfrak{F}_{\mathcal{B}}(\Phi)=\varphi$. If $\varphi$ is an isomorphism, then so is $\Phi$.

Proof of Theorem 5.17. We will explicitly define a functor from the category of exact $\mathcal{B}$-modules to the category of exact real-rank-zero-like $\mathcal{S} \mathcal{T}$-modules.

Let an exact $\mathcal{B}$-module $N$ be given. We will define an $\mathcal{S} \mathcal{T}$-module $M$. We begin in the obvious way: For $x \in X$, let $M(\overline{\{x\}}, 1)=N\left(\bar{x}_{1}\right)$ and $M(\widetilde{\{x\}}, 0)=N\left(\widetilde{x}_{0}\right)$. Similarly, for $x \rightarrow y$, we define the even component of $i \frac{\widetilde{\{y\}}}{\{x\}}$ to be $i_{\widetilde{x}_{0}}$, the odd component of $r \frac{\overline{\{y\}}}{\{x\}}$ to be $r_{\bar{x}_{1}}^{\bar{y}_{1}}$, and the odd-to-even component of $\delta \frac{\widetilde{\{x\}}}{\{y\}}$ to be $\delta \bar{y}_{1}$. This makes sure that, finally, we will have $\mathfrak{F}_{\mathcal{B}}(M)=N$. Also, we of course define $\delta_{C}^{U}: M(C, 0) \rightarrow M(U, 1)$ to be zero for every boundary pair $(U, C)$ so that $M$ will be real-rank-zero-like.

For $x \geq y$, let $x \rightarrow x_{1} \rightarrow x_{2} \rightarrow \cdots \rightarrow x_{n} \rightarrow y$ be the unique path from $x$ to $y$. Define the even component of $i \frac{\overline{\{y\}}}{\{x\}}$ to be the composition $i_{\widetilde{x_{0}}}^{\widetilde{x_{10}}} i_{\widetilde{x_{10}}}^{\widetilde{x_{10}}} \cdots i i_{\widetilde{x_{0} 0}}$ and the odd component of $r \frac{\overline{\{y\}}}{\{x\}}$ as the composition $r_{\bar{x}_{1}}^{\overline{x_{1}}} r_{\overline{x_{1}}}^{\overline{x_{2}}} \cdots r \frac{\bar{y}_{1}}{x_{n_{1}}}$. In case of $x=y$, this specifies to $i_{\widetilde{x}_{0}}^{\widetilde{x}_{0}}=\mathrm{id}_{M(\widetilde{\{x\}}, 0)}$ and $r_{\bar{x}_{1}}^{\bar{x}_{1}}=\mathrm{id}_{M(\overline{\{x\}}, 1)}$. If we have $x \rightarrow y$, then these definitions coincide with the ones we gave before.

We observe that the groups $M(\widetilde{\{x\}}, 0)$ with the maps $i \frac{\widetilde{\{y\}}}{\{x\}}$ define a precosheaf on $\mathbb{B}=\{\widetilde{\{x\}} \mid x \in X\}$. By Lemma 4.8 it is in fact a cosheaf. We can therefore apply Lemma 4.7 and obtain groups $M(U, 0)$ for all sets $U$ and maps $i_{U}^{V}: M(U, 0) \rightarrow$ $M(V, 0)$ for open sets $U \subseteq V$ which fulfill the relations (1) and (3) in Proposition 3.3

For an arbitrary locally closed subset $Y \in \mathbb{L} \mathbb{C}(X)$ we write $Y=V \backslash U$ with open sets $U \subseteq V$ and define $M(Y, 0)$ as the cokernel of the map $i_{U}^{V}: M(U, 0) \rightarrow M(V, 0)$. 
That this definition does not depend on the choice of $U$ and $V$ can be seen in a way similar to the proof of [20, Lemma 2.15] using that pushouts of abelian groups preserve cokernels. We obtain maps $r_{V}^{Y}: M(V, 0) \rightarrow M(Y, 0)$ for every open set $V$ with relatively closed subset $Y \subseteq V$ such that the following holds: If $Y \in \mathbb{L}(X)$ can be written as differences $V_{i} \backslash U_{i}$ of open sets for $i \in\{1,2\}$ such that $U_{1} \subseteq U_{2}$ and $V_{1} \subseteq V_{2}$, then the diagram

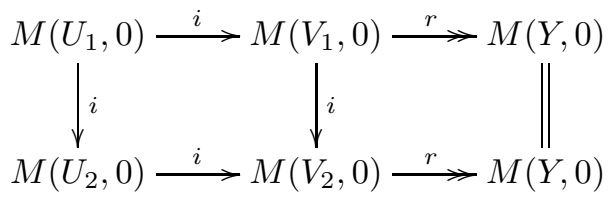

commutes.

For a relatively open subset $U \subseteq Y \in \mathbb{L} \mathbb{C}(X)$ we obtain a unique map $i_{U}^{Y}: M(U, 0) \rightarrow$ $M(Y, 0)$ using the diagram

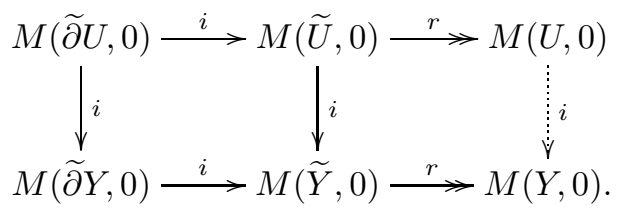

It is easy to check that this map coincides with the previously defined one in case $Y$ is open.

We find that, for $Y_{i} \in \mathbb{L} \mathbb{C}(X)$ with $Y_{1} \subseteq Y_{2}$ open, and $Y_{i}=V_{i} \backslash U_{i}$ for $i \in\{1,2\}$ and open sets $U_{i}, V_{i}$ such that $U_{1} \subseteq U_{2}$ and $V_{1} \subseteq V_{2}$, the diagram

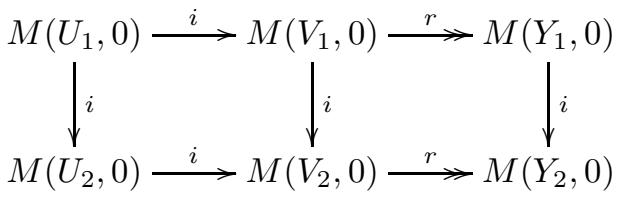

commutes. We know this already for the left-hand square. For the right-hand square, it can be seen as follows: since $V_{1}$ is covered by $U_{1}$ and $\widetilde{Y_{1}}$, it suffices to check commutativity on the images $i_{U_{1}}^{V_{1}}\left(M\left(U_{1}, 0\right)\right)$ and $i{\widetilde{Y_{1}}}_{V_{1}}\left(M\left(\widetilde{Y_{1}}, 0\right)\right)$. On $i_{U_{1}}^{V_{1}}\left(M\left(U_{1}, 0\right)\right)$ both compositions vanish. On the image of $M\left(\widetilde{Y_{1}}, 0\right)$, commutativity follows from (5.20) and (5.21) considering the diagram

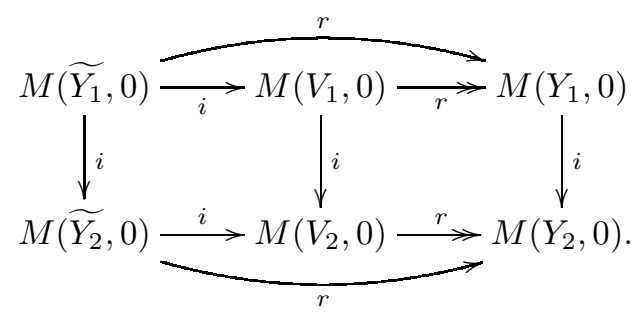


Now let $Y \in \mathbb{L} \mathbb{C}(X)$, let $U$ be a relatively open subset of $Y$ and let $C=Y \backslash U$. Consider the diagram

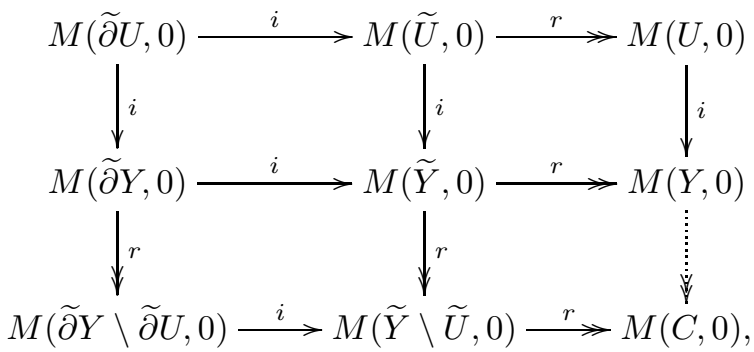

whose solid squares commute and whose rows and solid columns are exact. A diagram chase shows that there is a unique surjective map $r_{Y}^{C}: M(Y, 0) \rightarrow M(C, 0)$, as indicated by the dotted arrow, making the bottom-right square commute and making the right-hand column exact at $M(Y, 0)$. Again, we can easily check that this map coincides with the previously defined one in case $Y$ is open.

We have now defined the even part of the module $M$ completely. It is straightforward to check the relations (3) and (4) in Proposition 3.3. We will now prove that the relation (5) holds as well.

For this purpose, fix $Y \in \mathbb{L} \mathbb{C}(X)$, let $U \subseteq Y$ be open and let $C \subseteq Y$ be closed. Consider the diagram

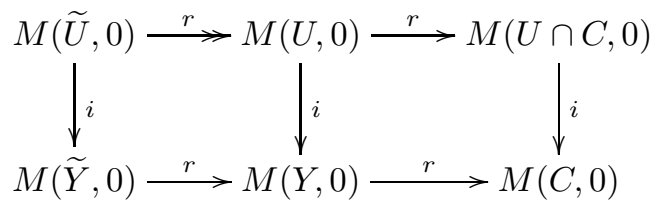

We would like to prove that the right-hand square commutes. The left-hand square commutes by definition of the map $i_{U}^{Y}$. Since $\widetilde{U} \cap C=U \cap C$, we can therefore assume without loss of generality that $U$ and $Y$ are open. Commutativity then follows from (5.22).

Next, we will convince ourselves that the relation $(2)$ in Proposition 3.3 holds on the even part of $M$. Let $W=Y \sqcup Z$ be a topologically disjoint union of subsets $Y, Z \in \mathbb{L}(X)$. Fix $w \in M(W, 0)$. Then $\left(w-w r_{W}^{Z} i_{Z}^{W}\right) r_{W}^{Z}=0$ as $i_{Z}^{W} r_{W}^{Z}=\operatorname{id}_{Z}$. Hence there is $y \in M(Y, 0)$ with $y i_{Y}^{W}=w-w r_{W}^{Z} i_{Z}^{W}$. Applying $r_{W}^{Y}$ shows $y=w r_{W}^{Y}$ as $i_{Z}^{W} r_{W}^{Y}=0$. We get

$$
w\left(r_{W}^{Y} i_{Y}^{W}+r_{W}^{Z} i_{Z}^{W}\right)=y i_{Y}^{W}+w r_{W}^{Z} i_{Z}^{W}=w .
$$

We have shown that $r_{W}^{Y} i_{Y}^{W}+r_{W}^{Z} i_{Z}^{W}=\operatorname{id}_{W}$ as desired.

We have defined all even groups for the desired module $M$ and the action of all transformations between them. We have checked all relations only involving transformations between even groups and verified exactness of $M(C, 0) \rightarrow M(Y, 0) \rightarrow$ $M(U, 0)$ for every boundary pair $Y=U \cup C$.

We intend to do the same for the odd part of the module $M$ in an analogous way. We start out with the given data consisting of the groups $M(\overline{\{x\}}, 1)$ and the maps $r_{\bar{x}_{1}}^{\bar{y}_{1}}, x \rightarrow y$, extend this to a sheaf on the basis $\{\overline{\{x\}} \mid x \in X\}$ of closed sets and apply Lemma 4.3 Observing that every locally closed subset of $X$ can be written as a difference of two nested closed sets and using the functoriality of the kernel of 
a group homomorphism, we define groups $M(\bar{Y}, 1)$ for all $Y \in \mathbb{L} \mathbb{C}(X)$ and actions for all transformations between these odd groups. Using arguments analogous to the ones above, we can verify the relations (1) to (5) in Proposition 3.3 on the odd part of $M$.

It remains to define the odd-to-even components of the boundary maps $\delta_{C}^{U}$ for all boundary pairs $(U, C)$, which has only been done in the special case $U=\widetilde{\{x\}}$, $C=\overline{\{y\}}$ with $x \rightarrow y$. Our general definition for $\delta_{C}^{U}: M(C, 1) \rightarrow M(U, 0)$ is

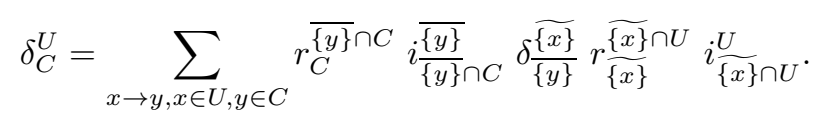

Our next aim is to verify the relations (6) (7) and (8) in Proposition 3.3. We begin with relation (6). Let $(U, C)$ be a boundary pair and let $C^{\prime} \subseteq C$ be relatively open. We have by the relations (3) and (5) that

$$
\begin{aligned}
& i_{C^{\prime}}^{C} \delta_{C}^{U}=i_{C^{\prime}}^{C}\left(\sum_{x \rightarrow y, x \in U, y \in C} r_{C}^{\overline{\{y\}} \cap C} i \overline{\{y\}} \cap \overline{\{y\}} \cap \overline{\frac{\{x\}}{\{y\}}} r \overline{\frac{\{x\}}{\{x\}}} \cap i \frac{U}{\{x\} \cap U}\right) \\
& =\sum_{x \rightarrow y, x \in U, y \in C} r_{C^{\prime}}^{\overline{\{y\}} \cap C^{\prime}} i \frac{\overline{y y\}}}{\{y\}} \cap C^{\prime} \delta \frac{\widetilde{\{x\}}}{\{y\}} r_{\{x\}}^{\widetilde{\{x\}}} \cap U i_{\{x\}}^{U} \cap .
\end{aligned}
$$

Since $C^{\prime}$ is relatively open in $C, \overline{\{y\}} \cap C^{\prime}$ is empty unless $y \in C^{\prime}$. Therefore, the above sum equals

$$
\delta_{C^{\prime}}^{U}=\sum_{x \rightarrow y, x \in U, y \in C^{\prime}} r_{C^{\prime}}^{\overline{\{y\}} \cap C^{\prime}} i \frac{\overline{\{y\}}}{\{y\}} \cap C^{\prime}, \widetilde{\{x\}} r \frac{\widetilde{\{x\}} \cap U}{\{x\}} i \frac{U}{\{x\} \cap U^{\prime}} .
$$

This shows relation $(6)$. The relation $(7)$ follows similarly.

Next we will check relation (8). Let $Y, Z, W \in \mathbb{L} \mathbb{C}(X)$ such that $Y \cup W \in \mathbb{L} \mathbb{C}(X)$ containing $Y, W$ as closed subsets, $Z \cup W \in \mathbb{L} \mathbb{C}(X)$ containing $Z, W$ as open subsets, and $W \subseteq Y \cup Z$. For each $x \in Z$ and $y \in Y$ with $x \rightarrow y$, we define $\gamma_{x, y}: M(Y, 1) \rightarrow$ $M(Z, 0)$ by

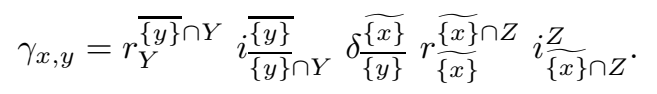

Since $W \backslash Y$ is an open subset of $Z$ (see the proof of Proposition 3.3), we have $\widetilde{\{x\}} \cap(W \backslash Y)=\widetilde{\{x\}} \cap Z$ for each $x \in W \backslash Y$. We also have $y \in W$ if $y \in Y$ satisfies $x \rightarrow y$ for some $x \in W \backslash Y$ because $W \subseteq Y \cup W$ is closed. Therefore, by the relation (4) we get

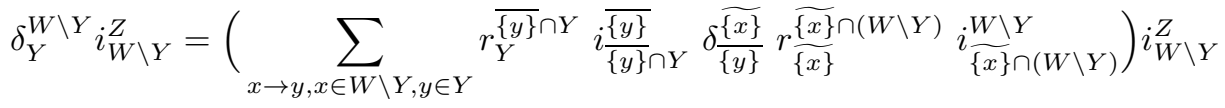

$$
\begin{aligned}
& =\sum_{x \rightarrow y, x \in W \backslash Y, y \in W \cap Y} r_{Y}^{\overline{\{y\}} \cap Y} i \frac{\overline{\{y\}}}{\{y\}} \cap Y \overline{\frac{\{x\}}{\{y\}}} r \frac{\widetilde{\{x\}} \cap Z}{\{x\}} i \frac{Z}{\{x\} \cap Z} \\
& =\sum_{(x, y) \in \Lambda_{1}} \gamma_{x, y}
\end{aligned}
$$

where we set

$$
\Lambda_{1}=\{(x, y) \mid x \rightarrow y, x \in W \backslash Y, y \in W \cap Y\}
$$


In a similar way using the facts that $W \backslash Z$ is a closed subset of $Y$ and that $Z$ is an open subset of $Y \cup Z$, we get

$$
r_{Y}^{W \backslash Z} \delta_{W \backslash Z}^{Z}=\sum_{(x, y) \in \Lambda_{2}} \gamma_{x, y}
$$

where we set

$$
\Lambda_{2}=\{(x, y) \mid x \rightarrow y, x \in W \cap Z, y \in W \backslash Z\}
$$

If we set

$$
\begin{aligned}
& \Lambda_{1}^{\prime}=\{(x, y) \mid x \rightarrow y, x \in W \cap Y \cap Z, y \in W \cap Y\} \\
& \Lambda_{2}^{\prime}=\{(x, y) \mid x \rightarrow y, x \in W \cap Z, y \in W \cap Y \cap Z\}
\end{aligned}
$$

then we have

$$
\{(x, y) \mid x \rightarrow y, x \in W \cap Z, y \in W \cap Y\}=\Lambda_{1} \sqcup \Lambda_{1}^{\prime}=\Lambda_{2} \sqcup \Lambda_{2}^{\prime}
$$

because $W \subseteq Y \cup Z$ implies $(W \cap Z) \backslash Y=W \backslash Y$ and $(W \cap Y) \backslash Z=W \backslash Z$. Therefore in order to show the equality $\delta_{Y}^{W \backslash Y} i_{W \backslash Y}^{Z}=r_{Y}^{W \backslash Z} \delta_{W \backslash Z}^{Z}$, it suffices to show

$$
\sum_{(x, y) \in \Lambda_{1}^{\prime}} \gamma_{x, y}=\sum_{(x, y) \in \Lambda_{2}^{\prime}} \gamma_{x, y}
$$

For each $p \in W \cap Y \cap Z$, we get

$$
\sum_{y \leftarrow p} r \overline{\frac{\{y\}}{\{p\}}} \delta \frac{\widetilde{\{p\}}}{\{y\}}=\sum_{x \rightarrow p} \delta \frac{\widetilde{\{x\}}}{\left\{\frac{\{p\}}{\{x\}}\right.}
$$

from the definition of $\mathcal{B}$-modules. Multiplying from the left with $r_{Y}^{\overline{\{p\}} \cap Y} i \frac{\overline{\{p\}}}{\{p\}} \cap Y$ and from the right with $r \frac{\widetilde{\{p\}} \cap Z}{\{p\}} i \frac{Z}{\{p\} \cap Z}$, and summing up over $p \in W \cap Y \cap Z$, we get

$$
\begin{aligned}
& \sum_{p \in W \cap Y \cap Z} r_{Y}^{\overline{\{p\}} \cap Y} i \frac{\overline{\{p\}}}{\{p\} \cap Y}\left(\sum_{y \leftarrow p} r \frac{\overline{\{y\}}}{\{p\}} \delta \frac{\overline{\{p\}}}{\{y\}}\right) r_{\frac{\{p\}}{\{p\}}}^{\widetilde{\{p}} i \frac{Z}{\{p\} \cap Z} \\
& =\sum_{p \in W \cap Y \cap Z} r_{Y}^{\overline{\{p\}} \cap Y} i_{\frac{\{p\}}{\{p\}} \cap Y}\left(\sum_{x \rightarrow p} \delta \frac{\widetilde{\{x\}}}{\{p\}} i \frac{\widetilde{\{p\}}}{\{x\}}\right) r \frac{\widetilde{\{p\}} \cap Z}{\{p\}} i \frac{Z}{\{p\} \cap Z} .
\end{aligned}
$$

By the relations $(3)$ and (4) we get

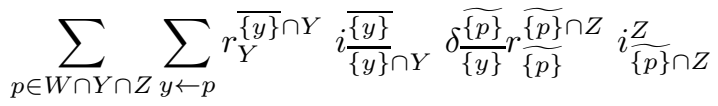

$$
\begin{aligned}
& =\sum_{p \in W \cap Y \cap Z} \sum_{x \rightarrow p} r_{Y}^{\overline{\{p\}} \cap Y} i \overline{\{p\}} \frac{\widetilde{\{x\}} \cap Y}{\left\{\frac{\{x\}}{\{p\}}\right.} r_{\frac{\{x\}}{\{x\}}} i \frac{Z}{\{x\} \cap Z} .
\end{aligned}
$$

Since $Y$ is locally closed, the conditions $p \in W \cap Y \cap Z, y \leftarrow p$ and $\overline{\{y\}} \cap Y \neq \emptyset$ imply $y \in Y$. This further implies $y \in W$ because $W \subseteq Y \cup W$ is closed. Hence the left-hand side of (5.25) equals $\sum_{(x, y) \in \Lambda_{1}^{\prime}} \gamma_{x, y}$. In a similar way, we can see that the right-hand side of (5.25) equals $\sum_{(x, y) \in \Lambda_{2}^{\prime}} \gamma_{x, y}$. Thus we have proven the relation (8), and this finishes the verification of all relations in Proposition 3.3 .

Hence, $M$ is indeed an $\mathcal{S} \mathcal{T}$-module. To see that $M$ is exact, it remains to show that the sequences $M(C, 1) \stackrel{\delta_{C}^{U}}{\longrightarrow} M(U, 0) \stackrel{i_{U}^{Y}}{\longrightarrow} M(Y, 0)$ and $M(Y, 1) \stackrel{r_{Y}^{C}}{\longrightarrow} M(C, 1) \stackrel{\delta_{C}^{U}}{\longrightarrow}$ $M(U, 0)$ are exact for all boundary pairs $(U, C)$ with $Y=U \cup C$. 
Fix an element $x \in X$ and consider the commutative diagram

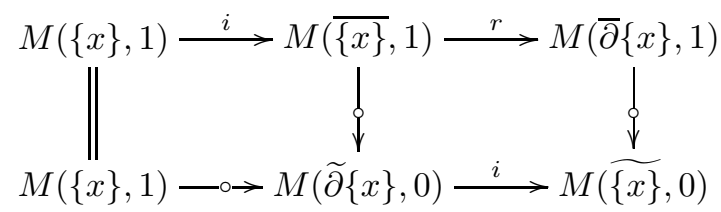

Using exactness of the upper row and the fact that $N$ was an exact $\mathcal{B}$-module, a diagram chase shows that the bottom row is exact. In a similar way, we see that the sequence

$$
M(\overline{\{x\}}, 1) \rightarrow M(\bar{\partial}\{x\}, 0) \rightarrow M(\{x\}, 0) .
$$

is exact for every $x \in X$.

Next, let $Y \in \mathbb{L} \mathbb{C}(X)$ and let $x \in Y$ be a closed point. Then $Y \cap \widetilde{\{x\}}$ is relatively closed in $\widetilde{\{x\}}$ because $Y$ is locally closed. A diagram chase in the commutative diagram

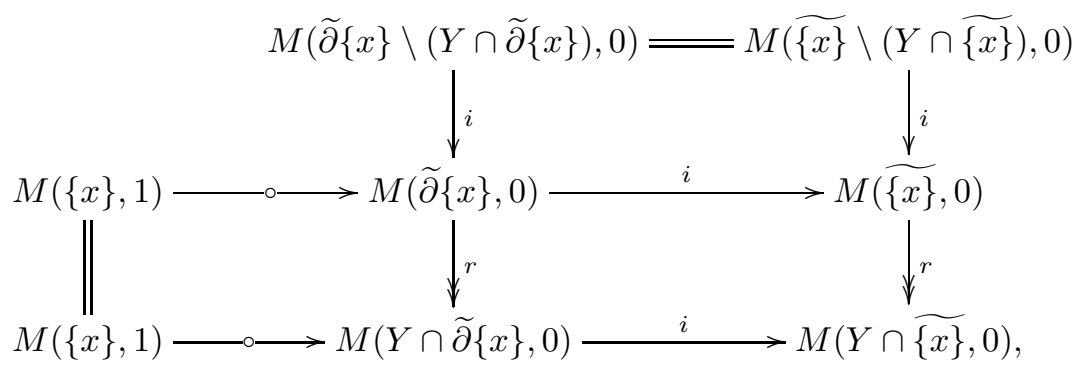

whose columns and top row are exact, yields exactness of the bottom row. By a diagram chase in the commutative diagram

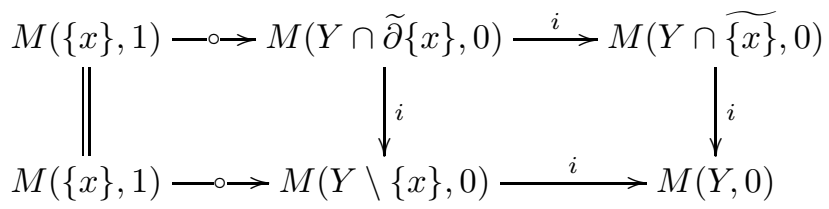

using the exact cosheaf sequence (4.6) for the covering $(Y \backslash\{x\}, Y \cap \widetilde{\{x\}})$ of $Y$ we obtain exactness of the bottom row. Notice that, using a further diagram chase, it is not hard to deduce the exactness of the cosheaf sequence for a relatively open covering of a locally closed set from the open case.

We have established the exactness of the sequence $M(C, 1) \stackrel{\delta_{C}^{U}}{\longrightarrow} M(U, 0) \stackrel{i_{U}^{Y}}{\longrightarrow}$ $M(Y, 0)$ for all boundary pairs $(U, C)$ with $C$ a singleton. Analogously, we find that $M(Y, 1) \stackrel{r_{Y}^{C}}{\longrightarrow} M(C, 1) \stackrel{\delta_{C}^{U}}{\longrightarrow} M(U, 0)$ is exact whenever $U$ is a singleton.

We will proceed by an inductive argument. Let $n \geq 1$ be a natural number and assume that exactness of the sequence $M(C, 1) \stackrel{\delta_{C}^{U}}{\longrightarrow} M(U, 0) \stackrel{i_{U}^{Y}}{\longrightarrow} M(Y, 0)$ is proven for all boundary pairs $(U, C)$ for which $C$ has at most $n$ elements. Let $(U, C)$ be a boundary pair such that $C$ has $n+1$ elements. Write $Y=U \cup C$. Let $p \in C$ be a maximal point and set $U^{\prime}=U \cup\{p\}, C^{\prime}=C \backslash\{p\}$. Then $\left(U^{\prime}, C^{\prime}\right)$ is a boundary 
pair. A diagram chase in the commutative diagram

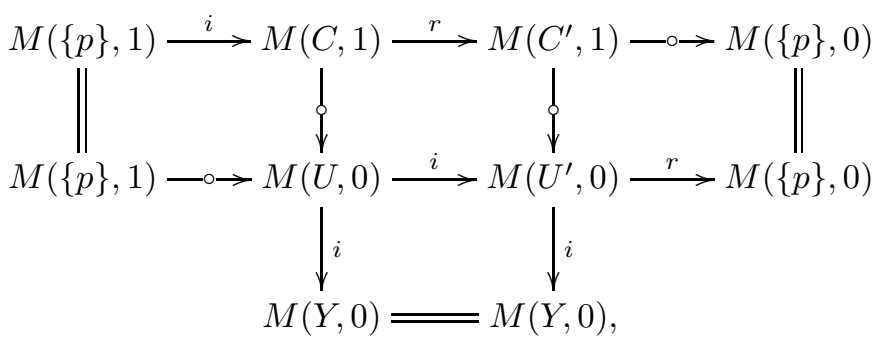

whose rows and third column are exact, shows exactness of the second column. Again, exactness of $M(Y, 1) \stackrel{r_{Y}^{C}}{\longrightarrow} M(C, 1) \stackrel{\delta_{C}^{U}}{\longrightarrow} M(U, 0)$ for all boundary pairs follows in a analogous manner. We conclude that $M$ is an exact $\mathcal{S T}$-module.

Summing up, we have associated an exact real-rank-zero-like $\mathcal{S} \mathcal{T}$-module with every exact $\mathcal{B}$-module. By a routine argument, this assignment extends uniquely to a functor $G$ from the category of exact $\mathcal{B}$-modules to the category of exact real-rank-zero-like $\mathcal{S} \mathcal{T}$-modules. Let $F$ be the restriction of the functor $\mathfrak{F}_{\mathcal{B}}$ to the category of exact real-rank-zero-like $\mathcal{S} \mathcal{T}$-modules. Then the composition $G F$ is equal to the identity functor on the category of exact $\mathcal{B}$-modules. It remains to show that $F G$ is naturally isomorphic to the identity functor on the category of exact real-rank-zero-like $\mathcal{S} \mathcal{T}$-modules.

Let $M$ be an exact real-rank-zero-like $\mathcal{S} \mathcal{T}$-module. We will construct a natural $\mathcal{S} \mathcal{T}$-module isomorphism $\eta_{M}: M \rightarrow(F G)(M)$. For $x \in X$ we have $M(\{x\}, 0)=$ $(F G)(M)(\widetilde{\{x\}}, 0)$ and $M(\overline{\{x\}}, 1)=(F G)(M)(\overline{\{x\}}, 1)$. Hence we set $\eta_{M}(\widetilde{\{x\}}, 0)=$ $\operatorname{id}_{M(\widetilde{\{x\}}, 0)}$ and $\eta_{M}(\overline{\{x\}}, 1)=\operatorname{id}_{M(\overline{\{x\}}, 1)}$. Using the universal property of kernels and cokernels we obtain natural group homomorphisms $f_{Y}: M(Y, 1) \rightarrow(F G)(M)(Y, 1)$ and $g_{Y}:(F G)(M)(Y, 0) \rightarrow M(Y, 0)$ for every $Y \in \mathbb{L} \mathbb{C}(X)$. An application of the Five Lemma shows that these are in fact isomorphisms. We can therefore define $\eta_{M}(Y, 1)=f_{Y}$ and $\eta_{M}(Y, 0)=\left(g_{Y}\right)^{-1}$.

Finally, we check that this collection of maps constitutes an $\mathcal{S T}$-module homomorphism, that is, the group homomorphism $\eta_{M}: M \rightarrow(F G)(M)$ intertwines the actions of the category $\mathcal{S T}$ on $M$ and on $(F G)(M)$. By construction this is true for the transformations $\left(i \frac{\widetilde{\{y\}}}{\{x\}}, 0\right),\left(r \frac{\overline{\{y\}}}{\{x\}}, 1\right)$ and $\delta \frac{\widetilde{\{x\}}}{\{y\}}$ for all $x, y \in X$ with $x \rightarrow y$. By Lemma 4.3 and Lemma 4.7 it is also true for the transformation $\left(i_{U}^{V}, 0\right)$ for all open subset $U, V$ of $X$ with $U \subseteq V$ and for $\left(r_{C}^{D}, 1\right)$ for all closed subsets $C, D$ of $X$ with $D \subseteq C$.

Let $V \subseteq X$ be open and let $Y \subseteq V$ be relatively closed. Since $\left(r_{V}^{Y}, 0\right)$ was defined as a natural projection onto a cokernel, our assertion holds for this transformation as well. Consequently, by (5.21) the assertion also follows for the transformation $\left(i_{U}^{Y}, 0\right)$ for $Y \in \mathbb{L} \mathbb{C}(X)$ and $U \subseteq Y$ relatively open. Finally (5.23) implies the assertion for the transformation $r_{Y}^{C}$ with $Y \in \mathbb{L} \mathbb{C}(X)$ and $C \subseteq Y$ relatively closed. We have shown that $\eta$ intertwines the actions of all even transformations on the 0 -parts of $M$ and $(F G)(M)$. By analogous arguments the same follows for the actions of all even transformations on the 1-parts of $M$ and $(F G)(M)$.

Our last step is to consider the action of a boundary transformation $\delta_{C}^{U}$ for a boundary pair $(U, C)$. Since $M$ and $(F G)(M)$ are real-rank-zero-like the 0-to-1 component of $\delta_{C}^{U}$ acts trivially on both modules. We have already seen that the 
assertion is true for the 1-to- 0 component of $\delta_{C}^{U}$ in the specific case that $(U, C)=$ $(\widetilde{\{x\}}, \overline{\{y\}})$ with $x \rightarrow y$. The general case then follows from (5.24) since $X$ is an EBP space.

\section{REDUCED FILTERED K-THEORY}

Let $X$ be an arbitrary finite $T_{0}$-space. We recall some definitions and facts from [3]. In [23], Gunnar Restorff introduced reduced filtered K-theory $\mathrm{FK}_{\mathcal{R}}$ and showed that it classifies purely infinite Cuntz-Krieger algebras up to stable isomorphism. In [3], the range of reduced filtered K-theory is established with respect to purely infinite Cuntz-Krieger algebras.

Definition 6.1 ([3, Definition 3.1]). Let $\mathcal{R}$ denote the universal pre-additive category generated by objects $x_{1}, \widetilde{\partial} x_{0}, \widetilde{x}_{0}$ for all $x \in X$ and morphisms $\delta_{x_{1}}^{\widetilde{\partial} x_{0}}$ and $i_{\widetilde{\partial} x_{0}}^{\widetilde{x}_{0}}$ for all $x \in X$, and $i \widetilde{y}_{\widetilde{y}_{0}}^{\widetilde{x} x_{0}}$ when $y \rightarrow x$, subject to the relations

$$
\begin{gathered}
\delta_{x_{1}}^{\widetilde{\partial} x_{0}} i_{\widetilde{\partial} x_{0}}^{\widetilde{x}_{0}}=0 \\
i_{p} i \frac{\widetilde{\partial} x_{0}}{y(p)_{0}}=i_{q} i \frac{\widetilde{\partial} x_{0}}{y(q)_{0}}
\end{gathered}
$$

for all $x \in X$, all $y \in X$ satisfying $y>x$, and all paths $p, q \in \operatorname{Path}(y, x)$, where for a path $p=\left(z_{k}\right)_{k=1}^{n}$ in $\operatorname{Path}(y, x)$, we define $y(p)=z_{2}$, and

$$
i_{p}=i_{\widetilde{z_{n}}}^{\widetilde{\partial} z_{n-1}} i_{\widetilde{\partial} z_{n-1}}^{\widetilde{z_{n-2}}} \ldots i_{\widetilde{z_{30}}}^{\widetilde{\partial} z_{20}} i_{\widetilde{\partial} z_{20}}^{\widetilde{z_{20}}}
$$

Definition 6.4. It is easy to see that the relations in $\mathcal{S T}$ corresponding to (6.2) and (6.3) hold. We can thus define an additive functor $\mathcal{R} \rightarrow \mathcal{S T}$ by $x_{1} \mapsto(\{x\}, 1)$, $\widetilde{\partial} x_{0} \mapsto(\widetilde{\partial}\{x\}, 0)$ and $\widetilde{x}_{0} \mapsto(\widetilde{\{x\}}, 0)$, and in the obvious way on morphisms. Let $\left.\mathfrak{F}_{\mathcal{R}}: \mathfrak{M o d}(\mathcal{S T}) \rightarrow \mathfrak{M} o d_{\mathcal{R}}\right)$ denote the induced functor. Define reduced filtered $\mathrm{K}$-theory, $\mathrm{FK}_{\mathcal{R}}$ as the composition of $\mathrm{FK}_{\mathcal{S} \mathcal{T}}$ with $\mathfrak{F}_{\mathcal{R}}$.

An equivalent definition of the functor $\mathrm{FK}_{\mathcal{R}}$ is given in [3, Definition 3.4].

Definition 6.5 ([3, Definition 3.6]). An $\mathcal{R}$-module $M$ is called exact if the sequences

$$
\begin{gathered}
M\left(x_{1}\right) \stackrel{\delta}{\rightarrow} M\left(\widetilde{\partial} x_{0}\right) \stackrel{i}{\rightarrow} M\left(\widetilde{x}_{0}\right) \\
\bigoplus_{(p, q) \in \mathrm{DP}(x)} M\left(\widetilde{s(p, q)_{0}}\right) \stackrel{\left(i_{p}-i_{q}\right)}{\longrightarrow} \bigoplus_{y \rightarrow x} M\left(\widetilde{y}_{0}\right) \stackrel{\left(i_{\tilde{y}_{0}}\right)}{\longrightarrow} M\left(\widetilde{\partial} x_{0}\right) \longrightarrow 0
\end{gathered}
$$

are exact for all $x \in X$, where $\mathrm{DP}(x)$ denotes the set of pairs of distinct paths $(p, q)$ to $x$ and from some common element which is denoted $s(p, q)$.

The following lemma is a generalization of [3, Lemma 3.9]. We omit the proof as the same technique applies here.

Lemma 6.8. Let $M$ be an exact real-rank-zero-like $\mathcal{S T}$-module. Let $Y$ be an open subset of $X$ and let $\left(U_{i}\right)_{i \in I}$ be an open covering of $Y$. Then the following sequence is exact:

$$
\bigoplus_{i, j \in I} M\left(U_{i} \cap U_{j}, 0\right) \stackrel{\left(_{U_{i} \cap U_{j}}^{U_{i}}-i_{U_{i} \cap U_{j}}^{U_{j}}\right)}{\longrightarrow} \bigoplus_{i \in I} M\left(U_{i}, 0\right) \stackrel{\left(i_{U_{i}}^{Y}\right)}{\longrightarrow} M(Y, 0) \longrightarrow 0 .
$$


Corollary 6.9. Let $M$ be an exact real-rank-zero-like $\mathcal{S T}$-module and set $N=$ $\mathfrak{F}_{\mathcal{R}}(M)$. Then $N$ is an exact $\mathcal{R}$-module.

Remark 6.10. If $X$ is a unique path space, then the set $\mathrm{DP}(x)$ is empty for every $x \in X$. Hence, for an exact $\mathcal{R}$-module $M$, the map $\left(i_{\widetilde{y}_{0}}^{\widetilde{\partial} x_{0}}\right): \bigoplus_{y \rightarrow x} M\left(\widetilde{y}_{0}\right) \rightarrow M\left(\widetilde{\partial} x_{0}\right)$ is an isomorphism. In this sense, the groups $M\left(\widetilde{\partial} x_{0}\right)$ are redundant for an exact $\mathcal{R}$-module in case $X$ is a unique path space.

By combining the following Proposition 6.11] and Theorem 6.12, one may obtain a complete description of the range of reduced filtered K-theory for purely infinite graph $C^{*}$-algebras and Cuntz-Krieger algebras.

Proposition 6.11 (3, Proposition 4.7]). Let $A$ be a purely infinite graph $C^{*}$-algebra over $X$. Then $\operatorname{FK}_{\mathcal{R}}(A)$ is an exact $\mathcal{R}$-module, and $\operatorname{FK}_{\{x\}}^{1}(A)$ is free for all $x \in X$.

If $A$ is a purely infinite Cuntz-Krieger algebra over $X$, then furthermore $\mathrm{K}_{1}(A(x))$ and $\mathrm{K}_{0}(A(\overline{\{x\}}))$ are finitely generated, and the rank of $\mathrm{K}_{1}(A(x))$ coincides with the rank of the cokernel of the map $i: \mathrm{K}_{0}\left(A(\widetilde{\partial}\{x\}) \rightarrow \mathrm{K}_{0}(A(\widetilde{\{x\}}))\right.$, for all $x \in X$.

Theorem 6.12 ([3, Theorem 4.8]). Let $M$ be an exact $\mathcal{R}$-module with $M\left(x_{1}\right)$ free for all $x \in X$. Then there exists a countable graph $E$ satisfying that all vertices in $E$ are regular and support at least two cycles, that $C^{*}(E)$ is tight over $X$ and that $\mathrm{FK}_{\mathcal{R}}\left(C^{*}(E)\right)$ is isomorphic to $M$. By construction $C^{*}(E)$ is purely infinite.

The graph $E$ can be chosen to be finite if (and only if) $M\left(x_{1}\right)$ and $M\left(\widetilde{x}_{0}\right)$ are finitely generated, and the rank of $M\left(x_{1}\right)$ coincides with the rank of the cokernel of $i: M\left(\widetilde{\partial} x_{0}\right) \rightarrow M\left(\widetilde{x}_{0}\right)$, for all $x \in X$. If $E$ is chosen finite, then by construction $C^{*}(E)$ is a Cuntz-Krieger algebra.

In Corollary 7.16, we combine this range-of-invariant theorem with the isomorphism lifting result from the next section.

\section{AN INTERMEDiATE INVARIANT}

In this section, we define one more invariant, which, in a sense, can be thought of as a union or join of reduced filtered K-theory $\mathrm{FK}_{\mathcal{R}}$ and filtered $\mathrm{K}$-theory restricted to canonical base $\mathrm{FK}_{\mathcal{B}}$. It functions as an intermediate invariant towards concrete filtered K-theory $\mathrm{FK}_{\mathcal{S T}}$.

Let $X$ be a unique path space.

Definition 7.1. Let $\mathcal{B R}$ denote the universal pre-additive category generated by objects $x_{1}, \bar{x}_{1}, \widetilde{x}_{0}$ for all $x \in X$ and morphisms $i_{x_{1}}^{\bar{x}_{1}}$ for all $x \in X$ and $r \bar{x}_{\bar{x}_{1}}, \delta_{\bar{y}_{1}}$ and $i_{\widetilde{x}_{0}}^{\widetilde{y}_{0}}$ when $x \rightarrow y$, subject to the relations

$$
\sum_{x \rightarrow y} r_{\bar{x}_{1}}^{\bar{y}_{1}} \delta_{\bar{y}_{1}}^{\widetilde{x}_{0}}=\sum_{z \rightarrow x} \delta_{\bar{x}_{1}} i_{\widetilde{z}_{0}} \widetilde{x}_{0}
$$

for all $x \in X$ and

$$
i_{x_{1}}^{\bar{x}_{1}} r_{\bar{x}_{1}}^{\bar{y}_{1}}=0
$$

when $x \rightarrow y$. 
As before, there is a canonical additive functor $\mathcal{B R} \rightarrow \mathcal{S T}$, inducing a functor $\mathfrak{F}_{\mathcal{B R}}: \mathfrak{M o d}(\mathcal{S T}) \rightarrow \mathfrak{M}_{\mathfrak{o d}}(\mathcal{B R})$. Define $\mathrm{FK}_{\mathcal{B R}}$ as the composition of $\mathrm{FK}_{\mathcal{S} \mathcal{T}}$ with $\mathfrak{F}_{\mathcal{B R}}$.

The category $\mathcal{B}$ embeds into the category $\mathcal{B R}$, and a forgetful functor $\mathfrak{M o d}(\mathcal{B R}) \rightarrow$ $\mathfrak{M o d}(\mathcal{B})$ is induced. We define an additive functor $\mathfrak{F}_{\mathcal{B R}, \mathcal{R}}: \mathfrak{M o d}(\mathcal{B R}) \rightarrow \mathfrak{M o d}(\mathcal{R})$ by

$$
M\left(\widetilde{\partial} x_{0}\right)=\bigoplus_{y \rightarrow x} M\left(\widetilde{y}_{0}\right)
$$

and $\delta_{x_{1}}^{\widetilde{\partial} x_{0}}=\left(i_{x_{1}}^{\bar{x}_{1}} \delta_{\bar{x}_{1}}^{\widetilde{y}_{0}}\right)$ and otherwise in the obvious way.

Definition 7.4. A $\mathcal{B R}$-module $M$ is called exact if the sequences

$$
\begin{gathered}
M\left(\bar{x}_{1}\right) \stackrel{\left(r_{\bar{x}_{1}}^{\bar{y}_{1}}-\delta_{\bar{x}_{1}}^{\widetilde{z}_{0}}\right)}{\longrightarrow} \bigoplus_{x \rightarrow y} M\left(\bar{y}_{1}\right) \oplus \bigoplus_{z \rightarrow x} M\left(\widetilde{z}_{0}\right) \stackrel{\left(\begin{array}{l}
\delta_{\bar{y}_{1}}^{\widetilde{x}_{0}} \\
i_{\widetilde{z}_{0}}^{x_{0}}
\end{array}\right)}{\longrightarrow} M\left(\widetilde{x}_{0}\right) \\
0 \rightarrow M\left(x_{1}\right) \stackrel{i_{x_{1}}^{\bar{x}_{1}}}{\longrightarrow} M\left(\bar{x}_{1}\right) \stackrel{\left(r_{\bar{x}_{1}}^{\bar{y}_{1}}\right)}{\longrightarrow} \bigoplus_{x \rightarrow y} M\left(\bar{y}_{1}\right)
\end{gathered}
$$

are exact for all $x \in X$.

Lemma 7.7. Let $M$ be an exact real-rank-zero-like $\mathcal{S} \mathcal{T}$-module. Then $\mathfrak{F}_{\mathcal{B} \mathcal{R}}(M)$ is an exact $\mathcal{B R}$-module.

Proof. The proof is similar to the proof of Lemma 5.10 .

Theorem 7.8. Assume that $X$ is a unique path space. Let $M$ and $N$ be exact $\mathcal{B R}$-modules with $M\left(x_{1}\right)$ and $N\left(x_{1}\right)$ free for all non-open points $x \in X$, and let $\varphi: \mathfrak{F}_{\mathcal{B R}, \mathcal{R}}(M) \rightarrow \mathfrak{F}_{\mathcal{B R}, \mathcal{R}}(N)$ be an $\mathcal{R}$-module homomorphism. Then there exists a (not necessarily unique) $\mathcal{B R}$-module homomorphism $\Phi: M \rightarrow N$ such that $\mathfrak{F}_{\mathcal{B R}, \mathcal{R}}(\Phi)=\varphi$. If $\varphi$ is an isomorphism then, by construction, so is $\Phi$.

Proof. For $x \in X$, we define $\Phi_{x_{1}}=\varphi_{x_{1}}$ and $\Phi_{\widetilde{x}_{0}}=\varphi_{\widetilde{x}_{0}}$. In the following, we will define $\Phi_{\bar{x}_{1}}$ by induction on the partial order of $X$ in a way such that the relations

$$
\begin{aligned}
& r \bar{x}_{\bar{x}_{1}}^{\bar{y}_{1}} \Phi_{\bar{y}_{1}}=\Phi_{\bar{x}_{1}} r \frac{\bar{y}_{\bar{x}_{1}}}{\bar{y}_{1}}, \\
& \delta_{\bar{x}_{1}}^{\widetilde{z}_{0}} \Phi_{\widetilde{z}_{0}}=\Phi_{\bar{x}_{1}} \delta_{\bar{x}_{1}}^{\widetilde{z}_{0}} \\
& i_{x_{1}}^{\bar{x}_{1}} \Phi_{\bar{x}_{1}}=\Phi_{x_{1}} i_{x_{1}}^{\bar{x}_{1}}
\end{aligned}
$$

hold for all $y$ with $x \rightarrow y$ and all $z$ with $z \rightarrow x$. For closed points $x \in X$, we set

$$
\Phi_{\bar{x}_{1}}=i_{x_{1}}^{\bar{x}_{1}} \varphi_{x_{1}}\left(i_{x_{1}}^{\bar{x}_{1}}\right)^{-1} .
$$

Here we have used that, by exactness of (7.6), $i_{x_{1}}^{\bar{x}_{1}}$ is invertible as there is no $y$ with $x \rightarrow y$. While the condition (7.9) is empty, (7.10) is guaranteed by $\varphi$ being an $\mathcal{R}$-module homomorphism, and (7.11) holds by construction.

Now fix an element $w \in X$ and assume that $\Phi_{\bar{x}_{1}}$ is defined for all $x<w$ in a way such that (7.9) and (7.10) hold. Using the exact sequence (7.6) and the 
freeness of $\bigoplus_{w \rightarrow x} M\left(\bar{w}_{1}\right)$, we can choose a free subgroup $V \subseteq M\left(\bar{w}_{1}\right)$ such that $M\left(\bar{w}_{1}\right)$ decomposes as an inner direct sum

$$
M\left(\bar{w}_{1}\right)=V \oplus M\left(w_{1}\right) \cdot i_{w_{1}}^{\bar{w}_{1}} .
$$

We will define $\Phi_{\bar{w}_{1}}$ by specifying the two restrictions $\left.\Phi_{\bar{w}_{1}}\right|_{V}$ and $\left.\Phi_{\bar{w}_{1}}\right|_{M\left(w_{1}\right) \cdot i_{w_{1}}^{\bar{w}_{1}}}$. Consider the diagram

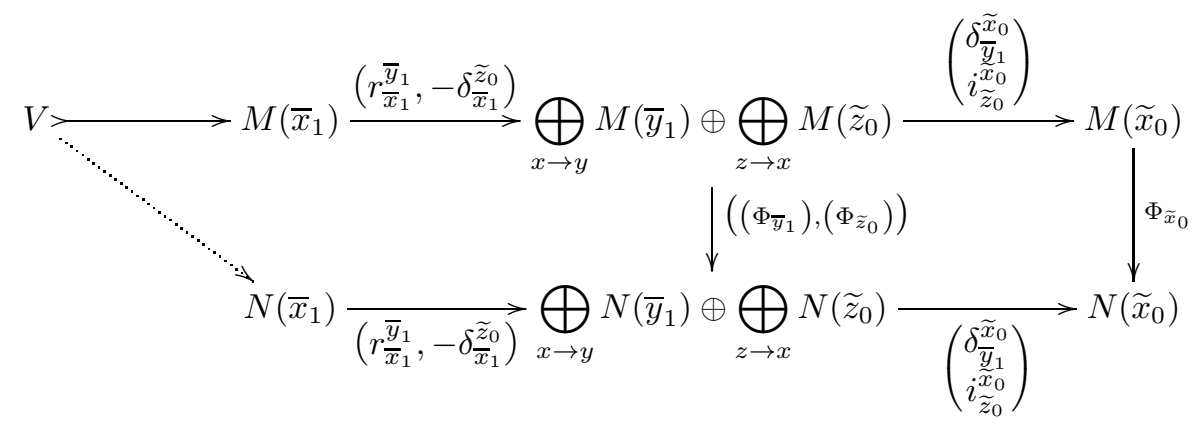

By assumption, the bottom row of this diagram is exact, the top row is exact in $\bigoplus_{x \rightarrow y} M\left(\bar{y}_{1}\right) \oplus \bigoplus_{z \rightarrow x} M\left(\widetilde{z}_{0}\right)$, and the right-hand square commutes. We can therefore choose a homomorphism $\left.\Phi_{\bar{x}_{1}}\right|_{V}: V \rightarrow N\left(\bar{x}_{1}\right)$ such that the left-hand pentagon commutes.

By exactness of (7.6), $i_{x_{1}}^{\bar{x}_{1}}$ is injective. Its corestriction onto its image $M\left(x_{1}\right) \cdot i_{x_{1}}^{\bar{x}_{1}}$ is thus an isomorphism. We may therefore define the restriction $\left.\Phi_{\bar{x}_{1}}\right|_{M\left(x_{1}\right) \cdot i_{x_{1}}^{\bar{x}_{1}}}$ in the unique way that makes the following diagram commute:

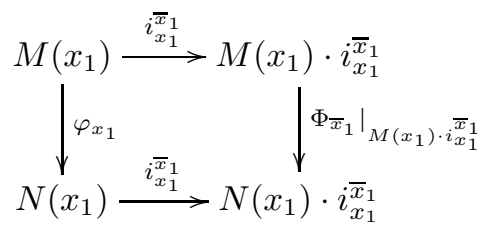

We have to check that $\Phi_{\bar{w}_{1}}=\left(\left.\Phi_{\bar{w}_{1}}\right|_{V},\left.\Phi_{\bar{w}_{1}}\right|_{M\left(w_{1}\right) \cdot i_{w_{1}}^{\bar{w}_{1}}}\right)$ fulfills (7.9) and (7.10) (with $x$ replaced with $w$ ). This is true on $V$ because of the commutativity of the lefthand side of (7.12). It is also true on the second summand: by (7.3), both sides of (7.9) vanish on this subgroup; (7.10) follows again from $\varphi$ being an $\mathcal{R}$-module homomorphism; and (7.11) holds by construction. This completes the induction step.

The claim, that $\Phi$ is an isomorphism whenever $\varphi$ is, follows from a repeated application of the Five Lemma.

Corollary 7.14. Assume that $X$ is an EBP space. Let $M$ and $N$ be exact, real-rank-zero-like $\mathcal{S T}$-modules with $M(\{x\}, 1)$ and $N(\{x\}, 1)$ free for all non-open points $x \in X$, and let $\varphi: \mathfrak{F}_{\mathcal{R}}(M) \rightarrow \mathfrak{F}_{\mathcal{R}}(N)$ be an $\mathcal{R}$-module homomorphism. Then there exists a (not necessarily unique) $\mathcal{S} \mathcal{T}$-module homomorphism $\Phi: M \rightarrow N$ satisfying $\mathfrak{F}_{\mathcal{R}}(\Phi)=\varphi$. If $\varphi$ is an isomorphism then, by construction, so is $\Phi$.

Proof. Combine Theorems 7.8 and 5.17 
Corollary 7.15. Let $A$ and $B$ be $C^{*}$-algebras of real rank zero over an EBP space $X$, and assume that $\mathrm{K}_{1}(A(x))$ and $\mathrm{K}_{1}(B(x))$ are free abelian groups for all non-open points $x \in X$. Then for any homomorphism $\varphi: \mathrm{FK}_{\mathcal{R}}(A) \rightarrow \mathrm{FK}_{\mathcal{R}}(B)$, there exist a (not necessarily unique) homomorphism $\Phi: \operatorname{FK}_{\mathcal{S T}}(A) \rightarrow \mathrm{FK}_{\mathcal{S} \mathcal{T}}(B)$ for which $\mathfrak{F}_{\mathcal{R}}(\Phi)=\varphi$. If $\varphi$ is an isomorphism then, by construction, so is $\Phi$.

Corollary 7.16. Let $A$ be a $C^{*}$-algebra over $X$ with real rank zero, and assume that $\mathrm{K}_{1}(A(x))$ is free for all $x \in X$. Then there exists a purely infinite graph $C^{*}$-algebra $C^{*}(E)$ that is tight over $X$ and satisfies $\mathrm{FK}_{\mathcal{R}}\left(C^{*}(E)\right) \cong \mathrm{FK}_{\mathcal{R}}(A)$. If $X$ is an EBP space, then automatically $\operatorname{FK}_{\mathcal{S T}}\left(C^{*}(E)\right) \cong \mathrm{FK}_{\mathcal{S T}}(A)$.

If furthermore for all $x \in X$, the group $\mathrm{K}_{*}(A(x))$ is finitely generated and $\operatorname{rank} \mathrm{K}_{1}(A(x))=\operatorname{rank} \mathrm{K}_{0}(A(x))$, then $C^{*}(E)$ can be chosen to be a purely infinite Cuntz-Krieger algebra.

Proof. Combine Theorem 6.12 with Corollary 6.9 and Corollary 7.15 ,

7.1. The particular case of the four-point space $\mathcal{D}$. Consider the space $\mathcal{D}=$ $\{1,2,3,4\}$ defined by $4 \rightarrow 3,4 \rightarrow 2,3 \rightarrow 1,2 \rightarrow 1$. The space $\mathcal{D}$ is not a unique path space. The second-named author showed in [6] that there exists a finite refinement FK $^{\prime}$ of filtered K-theory FK given by adding a $C^{*}$-algebra $R_{1 \backslash 4}$ over $\mathcal{D}$ to the collection $\left(R_{Y}\right)_{Y \in \mathbb{L} \mathbb{C}(\mathcal{D})^{*}}$ of representing objects, creating a larger category $\mathcal{N} \mathcal{T}^{\prime}$. By [6, Theorem 6.2.14], isomorphisms on the refined filtered K-theory FK' lift to $\operatorname{KK}(\mathcal{D})$-equivalences, and thereby (using [17]) to $\mathcal{D}$-equivariant ${ }^{*}$-isomorphisms, for stable Kirchberg $\mathcal{D}$-algebras with all simple subquotients in the bootstrap class. However, there exist two non-isomorphic stable Kirchberg $\mathcal{D}$-algebras $A$ and $B$ with real rank zero and simple subquotients in the bootstrap class such that $\mathrm{FK}(A) \cong$ $\mathrm{FK}(B)$, see [4, 6].

Proposition 7.17. Let $A$ and $B$ be $C^{*}$-algebras over $\mathcal{D}$, assume that $A$ and $B$ have real rank zero, and assume that $\mathrm{K}_{1}(A(x))$ and $\mathrm{K}_{1}(B(x))$ are free abelian groups for all $x \in\{1,2,3\}$. Then any homomorphism $\varphi: \operatorname{FK}_{\mathcal{R}}(A) \rightarrow \mathrm{FK}_{\mathcal{R}}(B)$ extends (nonuniquely) to a homomorphism $\Phi: \mathrm{FK}^{\prime}(A) \rightarrow \mathrm{FK}^{\prime}(B)$. If $\varphi$ is an isomorphism, then $\Phi$ is by construction an isomorphism.

Proof. By Section 6.2.5 of [6], the refined filtered K-theory FK' consists of the following groups and maps:

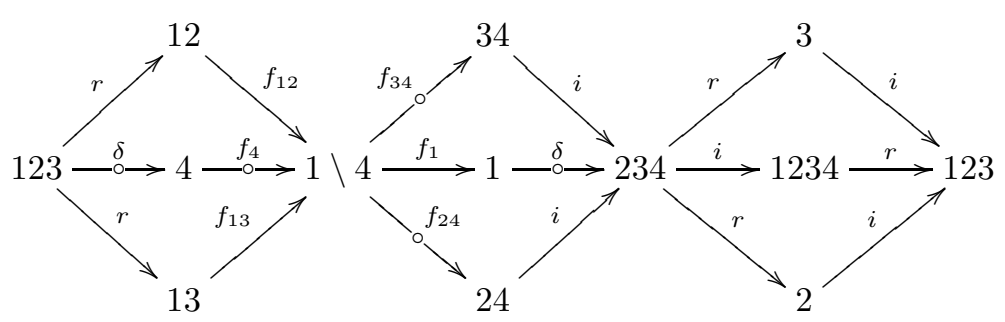


The proof of [4, Lemma 3.4] applies to the space $\mathcal{D}$, hence the two triangles
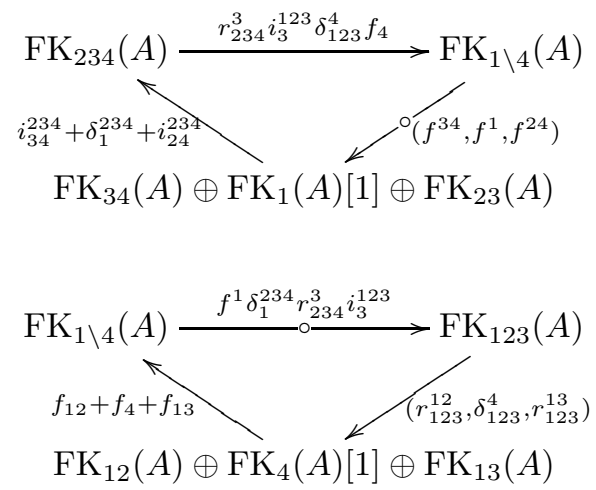

are exact. Since $A$ is of real rank zero, the maps

$$
\begin{gathered}
\mathrm{FK}_{123}^{0}(A) \stackrel{\delta_{123}^{4}}{\longrightarrow} \mathrm{FK}_{4}^{1}(A), \quad \mathrm{FK}_{12}^{0}(A) \stackrel{\delta_{12}^{34}}{\longrightarrow} \mathrm{FK}_{34}^{1}(A), \\
\mathrm{FK}_{13}^{0}(A) \stackrel{\delta_{13}^{24}}{\longrightarrow} \mathrm{FK}_{24}^{1}(A), \quad \mathrm{FK}_{1}^{0}(A) \stackrel{\delta_{1}^{234}}{\longrightarrow} \mathrm{FK}_{234}^{1}(A)
\end{gathered}
$$

vanish by Proposition 4 of [18. So for $C^{*}$-algebras over $\mathcal{D}$ of real rank zero, the invariant $\mathrm{FK}^{\prime}$ with the group $1 \backslash 4_{0}$ and its related maps omitted, consists of the following groups and maps:

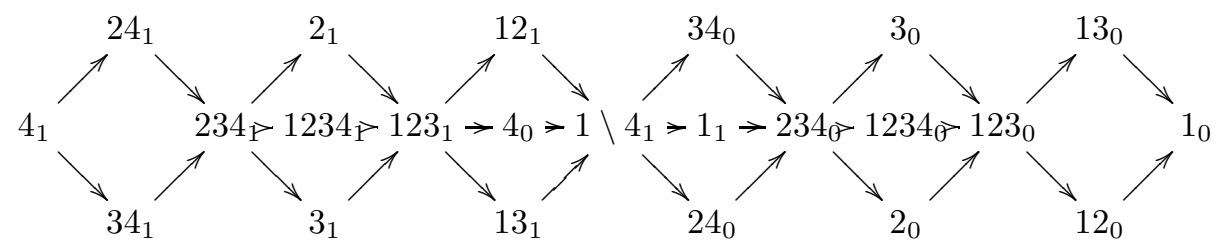

The reduced filtered K-theory $\mathrm{FK}_{\mathcal{R}}$ consists of the sequences $3_{1} \rightarrow 4_{0} \rightarrow 34_{0}$, $2_{1} \rightarrow 4_{0} \rightarrow 24_{0}, 1_{1} \rightarrow 234_{0} \rightarrow 1234_{0}$ together with the maps $34_{0} \rightarrow 234_{0}$ and $24_{0} \rightarrow 234_{0}$ and the group $4_{1}$.

We will now construct $\Phi=\left(\Phi_{Y}^{*}\right)_{Y \in \mathbb{L} \mathbb{C}(\mathcal{D})^{*} \cup\{1 \backslash 4\}}$ from $\varphi$. Define $\Phi_{\{x\}}^{1}=\varphi_{x_{1}}$, $\Phi_{\widetilde{\partial} x}^{0}=\varphi_{\widetilde{\partial} x_{0}}$, and $\Phi_{\{x\}}^{0}=\varphi_{\widetilde{x}_{0}}$ for all $x \in \mathcal{D}$. For $Y \in\{3,2,123,13,12,1\}$, the maps $\Phi_{Y}^{0}$ are constructed as the induced maps on cokernels, as in the proof of Theorem 5.17 .

Since $\operatorname{FK}_{1}^{1}(A)$ is free and the sequence

$$
0 \longrightarrow \mathrm{FK}_{4}^{0}(A) \stackrel{f_{4}}{\longrightarrow} \mathrm{FK}_{1 \backslash 4}^{1}(A) \stackrel{f^{1}}{\longrightarrow} \mathrm{FK}_{1}^{1}(A) \longrightarrow 0
$$

is exact, we can find a free subgroup $V_{1 \backslash 4}$ of $\operatorname{FK}_{1 \backslash 4}^{1}(A)$ for which $\operatorname{im} f_{4} \oplus V_{1 \backslash 4}=$ $\mathrm{FK}_{1 \backslash 4}^{1}(A)$. Consider the commuting diagram

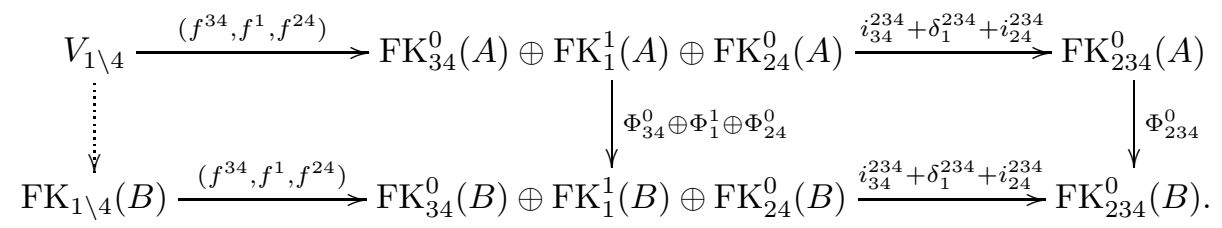


Since the bottom row is exact and the top row is a complex, and due to freeness of $V_{1 \backslash 4}$, we may choose a map $\psi: V_{1 \backslash 4} \rightarrow \mathrm{FK}_{1 \backslash 4}^{1}(B)$ that makes the left square of the diagram commute. Define $\Phi_{1 \backslash 4}^{1}$ on $\operatorname{im} f_{4} \oplus V_{1 \backslash 4}$ as $\Phi_{4}^{0}+\psi$. By construction,

$$
\Phi_{1 \backslash 4}^{1} f_{4}=f_{4} \Phi_{4}^{0}, \quad f^{1} \Phi_{1 \backslash 4}^{1}=\Phi_{1}^{1} f^{1}, \quad f^{34} \Phi_{1 \backslash 4}^{1}=\Phi_{34}^{0} f^{34}, \quad f^{24} \Phi_{1 \backslash 4}^{1}=\Phi_{24}^{0} f^{24},
$$

and by the Five Lemma, the homomorphism $\Phi_{1 \backslash 4}^{1}$ is an isomorphism if $\varphi$ is an isomorphism.

Similarly, to construct $\Phi_{12}^{1}$, use exactness of the sequence

$$
0 \longrightarrow \mathrm{FK}_{2}^{1}(A) \stackrel{i_{2}^{12}}{\longrightarrow} \mathrm{FK}_{12}(A) \stackrel{r_{12}^{1}}{\longrightarrow} \mathrm{FK}_{1}^{1}(A) \stackrel{\delta_{1}^{2}}{\longrightarrow} \mathrm{FK}_{2}^{0}(A)
$$

and freeness of $\mathrm{FK}_{1}^{1}(A)$ to choose a free subgroup $V_{12}$ of $\mathrm{FK}_{12}^{1}(A)$ for which $\operatorname{im} i_{2}^{12} \oplus$ $V_{12}=\mathrm{FK}_{12}^{1}(A)$. Consider the commuting diagram

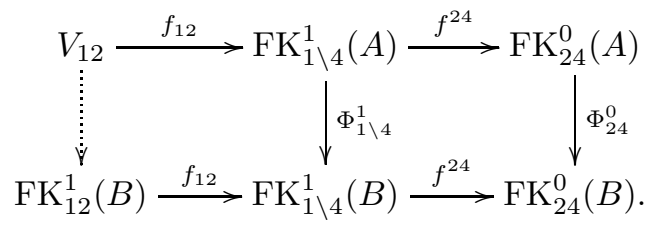

Using exactness of the bottom row and that the top row is a complex, the map $\Phi_{12}^{1}$ can be constructed so that

$$
\Phi_{12}^{1} i_{2}^{12}=i_{2}^{12} \Phi_{2}^{1}, \quad f_{12} \Phi_{12}^{1}=\Phi_{1 \backslash 4}^{1} f_{12} .
$$

Again due to the Five Lemma, $\Phi_{12}^{1}$ is an isomorphism if $\varphi$ is. The maps $\Phi_{13}^{1}, \Phi_{123}^{1}$, $\Phi_{1234}^{1}, \Phi_{234}^{1}, \Phi_{34}^{1}$, and $\Phi_{24}^{1}$ are constructed similarly and in the specified order.

Finally, the group $\mathrm{FK}_{1 \backslash 4}^{0}(A)$ is naturally isomorphic to

$$
\begin{aligned}
& \operatorname{coker}\left(\mathrm{FK}_{123}^{0}(A) \stackrel{\left(r_{123}^{12}, \delta_{123}^{4}, r_{123}^{13}\right)}{\longrightarrow} \mathrm{FK}_{12}^{0}(A) \oplus \mathrm{FK}_{4}^{1}(A) \oplus \mathrm{FK}_{13}^{1}(A)\right) \\
& =\mathrm{FK}_{4}^{1}(A) \oplus \operatorname{coker}\left(\mathrm{FK}_{123}^{0}(A) \stackrel{\left(r_{123}^{12}, r_{123}^{13}\right)}{\longrightarrow} \mathrm{FK}_{12}^{0}(A) \oplus \mathrm{FK}_{13}^{1}(A)\right)
\end{aligned}
$$

whose second summand, due to real rank zero, is naturally isomorphic to $\operatorname{FK}_{1}^{0}(A)$ Therefore, by defining $\Phi_{1 \backslash 4}^{0}$ as the map induced by $\Phi_{4}^{1} \oplus \Phi_{1}^{0}, \Phi$ becomes a $\mathcal{N} \mathcal{T}^{\prime}$ morphism.

Corollary 7.18. Let $A$ and $B$ be $C^{*}$-algebras over $\mathcal{D}$. Assume that $A$ and $B$ have real rank zero, that $\mathrm{K}_{1}(A(x))$ and $\mathrm{K}_{1}(B(x))$ are free abelian groups for all $x \in\{1,2,3\}$, and that $A$ and $B$ are in the bootstrap class of Meyer-Nest. Then any isomorphism $\mathrm{FK}_{\mathcal{R}}(A) \rightarrow \mathrm{FK}_{\mathcal{R}}(B)$ lifts to a $\mathrm{KK}(\mathcal{D})$-equivalence.

Proof. Combine Proposition 7.17 with [6, Theorem 6.2.14].

Corollary 7.19. Let $A$ and $B$ be stable Kirchberg $\mathcal{D}$-algebras of real rank zero, assume that $\mathrm{K}_{1}(A(x))$ and $\mathrm{K}_{1}(B(x))$ are free abelian groups for all $x \in\{1,2,3\}$, and assume that $A(x)$ and $B(x)$ are in the bootstrap class for all $x \in \mathcal{D}$. Then any isomorphism $\operatorname{FK}_{\mathcal{R}}(A) \rightarrow \mathrm{FK}_{\mathcal{R}}(B)$ lifts to a D -equivariant ${ }^{*}$-isomorphism $A \rightarrow B$.

Proof. Combine Proposition 7.17 with [6, Theorem 6.2.15]. 


\section{UNitAl FILTERED K-THEORY}

In [24, 2.1], Gunnar Restorff and Efren Ruiz showed that if a functor $F$ (that factors through the functor $\mathrm{K}_{0}$ ) strongly classifies a certain type of class of $C^{*}$-algebras up to stable isomorphism, then the functor $A \mapsto\left(F(A),\left[1_{A}\right] \in \mathrm{K}_{0}(A)\right)$ classifies unital, properly infinite $C^{*}$-algebras in the class up to isomorphism. A version with slightly generalized assumptions of this so-called meta-theorem may be found in [14] as Theorem 3.3. With these generalized assumptions, the theorem applies to filtered K-theory $\mathrm{FK}$ over accordion spaces $X$ with respect to Kirchberg $X$-algebras with simple subquotients in the bootstrap class.

Let $X$ be an arbitrary finite $T_{0}$-space. For $x, x^{\prime} \in X$, we let $\inf \left(x, x^{\prime}\right)$ denote the set $\left\{y \in X \mid y \rightarrow x, y \rightarrow x^{\prime}\right\}$.

Definition 8.1. The category $\mathfrak{M o d}(\mathcal{S T})^{\text {pt }}$ of pointed $\mathcal{S T}$-modules is defined to have objects $(M, m)$ where $M$ is a $\mathcal{S} \mathcal{T}$-module and $m \in M(X, 0)$, and morphisms $\varphi:(M, m) \rightarrow(N, n)$ that are $\mathcal{S} \mathcal{T}$-morphisms with $\varphi(m)=n$.

The category $\mathfrak{M o d}(\mathcal{B})^{\mathrm{pt}}$ of pointed $\mathcal{B}$-modules is defined similarly with objects $(M, m)$ where $M$ is a $\mathcal{B}$-module and

$$
m \in \operatorname{coker}\left(\bigoplus_{x, x^{\prime} \in X, y \in \inf \left(x, x^{\prime}\right)} M\left(\widetilde{y}_{0}\right) \stackrel{\left(i_{\widetilde{y}_{0}}^{\widetilde{x}_{0}}-i_{\widetilde{y}_{0}}^{\widetilde{x}_{0}^{\prime}}\right)}{\longrightarrow} \bigoplus_{x \in X} M\left(\widetilde{x}_{0}\right)\right)
$$

and a morphism $\varphi:(M, m) \rightarrow(N, n)$ is a $\mathcal{B}$-morphism whose induced map on the cokernels sends $m$ to $n$.

Similarly, the categories $\mathfrak{M o d}(\mathcal{B R})^{\mathrm{pt}}$ and $\mathfrak{M o d}(\mathcal{R})^{\mathrm{pt}}$ of pointed $\mathcal{B} \mathcal{R}$-modules respectively pointed $\mathcal{R}$-modules are defined.

Definition 8.2. A pointed $\mathcal{S} \mathcal{T}$-module $(M, m)$ is called exact if $M$ is an exact $\mathcal{S T}$-module, and real-rank-zero-like if $M$ is real-rank-zero-like. Similary, a pointed $\mathcal{B}$-module, $\mathcal{B R}$-module, or $\mathcal{R}$-module $(M, m)$ is called exact if $M$ is exact.

Lemma 8.3. Let $M$ be an exact real-rank-zero-like $\mathcal{S} \mathcal{T}$-module. Then the sequence

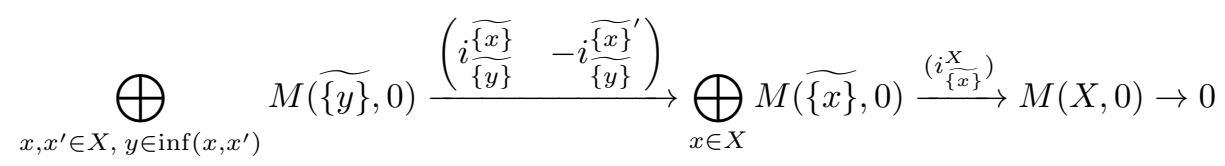

is exact.

Proof. By Lemma 6.8 the horizontal row of the following commuting diagram is exact:

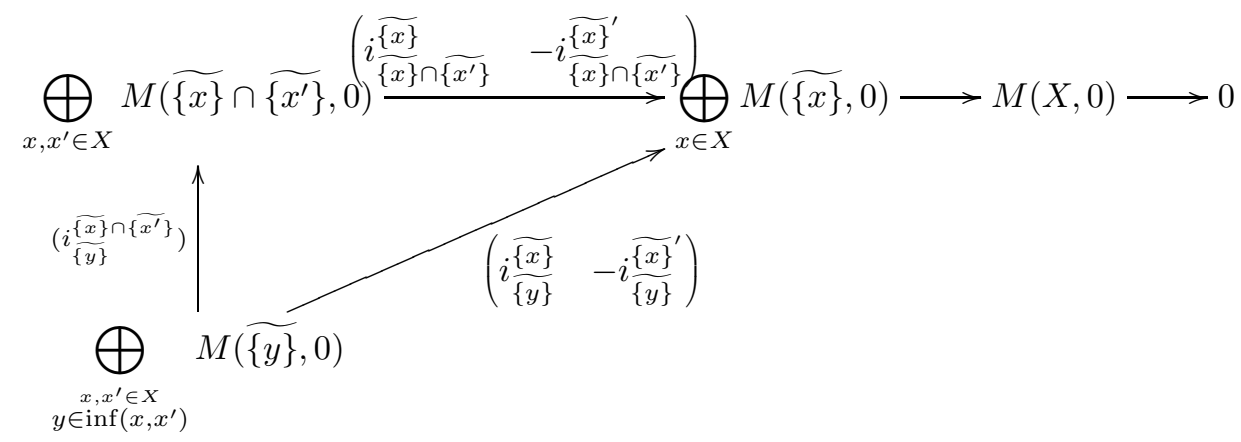


Furthermore, since for any pair $x, x^{\prime} \in X$ the collection $(\widetilde{\{y\}})_{y \in \inf \left(x, x^{\prime}\right)}$ covers $\widetilde{\{x\}} \cap$ $\widetilde{\left\{x^{\prime}\right\}}$, we see by Lemma 6.8 that the vertical map in the diagram is surjective. This establishes the desired result.

Definition 8.4. Let $A$ be a unital $C^{*}$-algebra over a finite $T_{0}$-space $X$. Its unital concrete filtered $\mathrm{K}$-theory $\operatorname{FK}_{\mathcal{S T}}^{\mathrm{unit}}(A)$ is defined as the pointed $\mathcal{S} \mathcal{T}$-module $\left(\mathrm{FK}_{\mathcal{S T}}(A),\left[1_{A}\right]\right)$.

If $A$ has real rank zero, then its unital reduced filtered $\mathrm{K}$-theory $\operatorname{FK}_{\mathcal{R}}^{\text {unit }}(A)$ is defined as the pointed $\mathcal{R}$-module $\left(\mathrm{FK}_{\mathcal{R}}(A), u(A)\right)$ where $u(A)$ is the unique element in

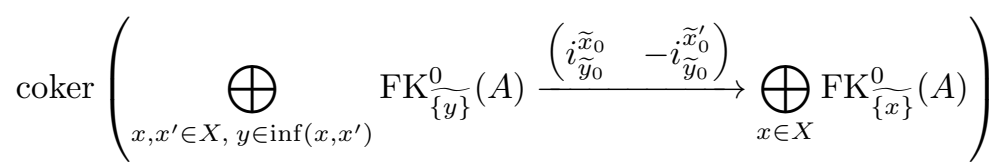

that is mapped to $\left[1_{A}\right]$ in $\mathrm{K}_{0}(A)$ by the map induced by the family $\left(\mathrm{FK}_{\{x\}}^{0}(A) \stackrel{i \frac{X}{[x\}}}{\longrightarrow}\right.$ $\left.\mathrm{FK}_{X}^{0}(A)\right)_{x \in X}$, see Lemma 8.3 .

If $A$ has real rank zero and $X$ is a unique path space, then its unital filtered $\mathrm{K}$-theory restricted to the canonical base $\operatorname{FK}_{\mathcal{B}}^{\text {unit }}(A)$ is defined similarly.

By Lemma 8.3, we may view the forgetful functor $\mathfrak{F}_{\mathcal{B}}: \mathfrak{M o d}(\mathcal{S T}) \rightarrow \mathfrak{M o d}(\mathcal{B})$ as a functor from pointed exact real-rank-zero-like $\mathcal{S} \mathcal{T}$-modules to pointed exact $\mathcal{B}$-modules and immediately obtain the following pointed version of Theorem 5.17

Proposition 8.5. For every EBP space $X$, the forgetful functor from exact pointed real-rank-zero-like $\mathcal{S} \mathcal{T}$-modules to exact pointed $\mathcal{B}$-modules is an equivalence of categories.

Proposition 8.6. Assume that $X$ is a unique path space. Let $(M, m)$ and $(N, n)$ be exact pointed $\mathcal{B R}$-modules with $M\left(x_{1}\right)$ and $N\left(x_{1}\right)$ free for all non-open points $x \in X$, and let $\varphi: \mathfrak{F}_{\mathcal{B R}, \mathcal{R}}(M) \rightarrow \mathfrak{F}_{\mathcal{B R}, \mathcal{R}}(N)$ be a pointed $\mathcal{R}$-module homomorphism. Then there exists a (not necessarily unique) pointed $\mathcal{B} \mathcal{R}$-module homomorphism $\Phi: M \rightarrow N$ satisfying $\mathfrak{F}_{\mathcal{B R}, \mathcal{R}}(\Phi)=\varphi$, and if $\varphi$ is an isomorphism, then $\Phi$ is by construction an isomorphism.

Proof. This follows from Theorem 7.8 since the groups $M\left(\widetilde{x}_{0}\right)$ are not forgotten by $\mathfrak{F}_{\mathcal{B R}, \mathcal{R}}$.

Corollary 8.7. Let $X$ be an accordion space, and let $A$ and $B$ be unital Kirchberg $X$-algebras of real rank zero with all simple subquotients in the bootstrap class. Then any isomorphism $\mathrm{FK}_{\mathcal{B}}^{\text {unit }}(A) \rightarrow \mathrm{FK}_{\mathcal{B}}^{\text {unit }}(B)$ lifts to an $X$-equivariant ${ }^{*}$-isomorphism $A \rightarrow B$.

Proof. This follows from Theorem 3.3 in [14 together with Theorem 3.11 and Corollary 5.19 .

Corollary 8.8. Let $X$ be an accordion space, and let $A$ and $B$ be unital Kirchberg $X$-algebras of real rank zero with all simple subquotients in the bootstrap class. Assume that $\mathrm{K}_{1}(A(x))$ and $\mathrm{K}_{1}(B(x))$ are free abelian groups for all $x \in X$. Then any isomorphism $\mathrm{FK}_{\mathcal{R}}^{\text {unit }}(A) \rightarrow \mathrm{FK}_{\mathcal{R}}^{\text {unit }}(B)$ lifts to an $X$-equivariant ${ }^{*}$-isomorphism $A \rightarrow B$. 
Proof. This follows from Theorem 3.3 in [14 together with Theorem 3.11 and Corollary 7.14

Remark 8.9. There exist, up to homeomorphism, precisely four contractible unique path spaces with four points that are not accordion spaces. For all these spaces, the categories $\mathcal{N} \mathcal{T}$ and $\mathcal{S} \mathcal{T}$ coincide. In [4, Gunnar Restorff, Efren Ruiz and the first-named author showed that if $X$ is one of these spaces, then FK is a complete invariant for stable Kirchberg $X$-algebras of real rank zero. Therefore, Corollaries 8.7 and 8.8 also hold for these spaces. Furthermore, the proof of Proposition 7.17 also applies to $\mathrm{FK}_{\mathcal{R}}^{\text {unit }}$ and unital $C^{*}$-algebras, hence Corollary 8.8 also holds for the space $\mathcal{D}$.

We now recall the unital version of the range result from [3].

Theorem 8.10 ([3, Theorem 5.5]). Let $X$ be a finite $T_{0}$-space, and let $(M, m)$ be an exact pointed $\mathcal{R}$-module. Assume that for all $x \in X, M\left(x_{1}\right)$ is a free abelian group,

$$
\operatorname{coker}\left(M\left(\widetilde{\partial} x_{0}\right) \stackrel{i_{\widetilde{x} x_{0}}^{\widetilde{x}_{0}}}{\longrightarrow} M\left(\widetilde{x}_{0}\right)\right)
$$

is finitely generated, and $\operatorname{rank} M\left(x_{1}\right) \leq \operatorname{rank} \operatorname{coker}\left(M\left(\widetilde{\partial} x_{0}\right) \stackrel{i_{\widetilde{\partial} x_{0}}^{\tilde{\tau}_{0}}}{\longrightarrow} M\left(\widetilde{x}_{0}\right)\right)$.

Then there exists a countable graph $E$ satisfying that all vertices in $E$ support at least two cycles, that $E^{0}$ is finite, that $C^{*}(E)$ is tight over $X$, and that $\mathrm{FK}_{\mathcal{R}}^{\text {unit }}\left(C^{*}(E)\right)$ is isomorphic to $(M, m)$. By construction $C^{*}(E)$ is unital and purely infinite.

The graph $E$ can be chosen to have only regular vertices if (and only if) the rank of $M\left(x_{1}\right)$ coincides with the rank of the cokernel of $i: M\left(\widetilde{\partial} x_{0}\right) \rightarrow M\left(\widetilde{x}_{0}\right)$ for all $x \in X$. If $E$ is chosen to have only regular vertices, then by construction $C^{*}(E)$ is a Cuntz-Krieger algebra.

Corollary 8.11. Let $X$ be a finite $T_{0}$-space and let $A$ be a unital $C^{*}$-algebra over $X$ of real rank zero. Assume for all $x \in X$ that $\mathrm{K}_{1}(A(x))$ is free, $\mathrm{K}_{0}(A(x))$ is finitely generated, and $\operatorname{rank} \mathrm{K}_{1}(A(x)) \leq \operatorname{rank} \mathrm{K}_{0}(A(x))$.

Then there exists a countable graph $E$ for which $C^{*}(E)$ is unital, purely infinite, and tight over $X$ such that $\mathrm{FK}_{\mathcal{R}}^{\text {unit }}\left(C^{*}(E)\right) \cong \mathrm{FK}_{\mathcal{R}}^{\text {unit }}(A)$. If $X$ is an EBP space, then automatically $\mathrm{FK}_{\mathcal{S} \mathcal{T}}^{\text {unit }}\left(C^{*}(E)\right) \cong \mathrm{FK}_{\mathcal{S} \mathcal{T}}^{\text {unit }}(A)$.

If furthermore $\operatorname{rank} \mathrm{K}_{1}(A(x))=\operatorname{rank} \mathrm{K}_{0}(A(x))$ for all $x \in X$, then $E$ can be chosen such that $C^{*}(E)$ is a purely infinite Cuntz-Krieger algebra.

Corollary 8.12. Let $X$ be an accordion space, and let $I \hookrightarrow A \rightarrow B$ be an extension of $C^{*}$-algebras. Assume that $A$ is unital and tight over $X$.

Then $A$ is a purely infinite Cuntz-Krieger algebra if and only if

- I is stably isomorphic to a purely infinite Cuntz-Krieger algebra,

- $B$ is a purely infinite Cuntz-Krieger algebra,

- the exponential map $\mathrm{K}_{0}(B) \rightarrow \mathrm{K}_{1}(I)$ vanishes.

Proof. Recall that Cuntz-Krieger algebras are purely infinite if and only if they have real rank zero. Assume that $A$ is a purely infinite Cuntz-Krieger algebra. It is well-known that then $B$ is also a purely infinite Cuntz-Krieger algebra and $I$ is stably isomorphic to one. By Theorem 4.2 of [21], $\mathrm{K}_{0}(B) \rightarrow \mathrm{K}_{1}(I)$ vanishes since $A$ has real rank zero and therefore is $\mathrm{K}_{0}$-liftable, see Remark 3.10 . 
Now, assume that $B$ is a purely infinite Cuntz-Krieger algebra, that $I$ is stably isomorphic to one, and that the map $\mathrm{K}_{0}(B) \rightarrow \mathrm{K}_{1}(I)$ vanishes. By Theorem 4.3 of [28, $A$ is $\mathcal{O}_{\infty}$-absorbing since $B$ and $I$ are. Since $B$ and $I$ are $\mathrm{K}_{0}$-liftable and $\mathrm{K}_{0}(B) \rightarrow \mathrm{K}_{1}(I)$ vanishes, $A$ is also $\mathrm{K}_{0}$-liftable (that is, $\mathrm{FK}(A)$ is real-rank-zerolike) by [7. Proposition 3.5]. So by pure infiniteness of $A$ it therefore follows from Theorem 4.2 of [21] that $A$ has real rank zero. For all $x \in X, \mathrm{~K}_{1}(A(x))$ is free since $B$ and $I$ are stably isomorphic to Cuntz-Krieger algebras. So by Theorem 8.10 there exists a real-rank-zero Cuntz-Krieger algebra $C$ that is tight over $X$ and has $\mathrm{FK}_{\mathcal{R}}^{\text {unit }}(A) \cong \mathrm{FK}_{\mathcal{R}}^{\text {unit }}(C)$. By Corollary 8.8 , $A$ and $C$ are isomorphic.

Remark 8.13. Corollary 8.12 holds in fact for all spaces $X$ for which $\mathrm{FK}_{\mathcal{R}}^{\text {unit }}$ is a complete invariant for unital Kirchberg $X$-algebras $A$ where $A(x)$ is in the bootstrap class and $\mathrm{K}_{1}(A(x))$ is free for all $x \in X$, see Remark 8.9

\section{ORDERED FILTERED K-THEORY}

The notion of ordered filtered K-theory was introduced by Søren Eilers, Gunnar Restorff, and Efren Ruiz in [13] to classify certain (not necessarily purely infinite) graph $C^{*}$-algebras of real rank zero. We hope that the results in this section will be useful for future work in this direction.

Recall that for a $C^{*}$-algebra $A$, a class in $\mathrm{K}_{0}(A)$ of the from $[p]_{0}$ for a projection $p$ in $M_{n}(A)$ for some $n \in \mathbb{N}$ is called positive. The positive cone $\mathrm{K}_{0}(A)^{+}$consists of all positive elements in $\mathrm{K}_{0}(A)$. For two $C^{*}$-algebras $A$ and $B$, a group homomorphism $\varphi: \mathrm{K}_{0}(A) \rightarrow \mathrm{K}_{0}(B)$ is called positive if $\varphi\left(\mathrm{K}_{0}(A)^{+}\right) \subseteq \mathrm{K}_{0}(B)^{+}$, and a group isomorphism $\varphi: \mathrm{K}_{0}(A) \rightarrow \mathrm{K}_{0}(B)$ is called an order isomorphism if $\varphi\left(\mathrm{K}_{0}(A)^{+}\right)=\mathrm{K}_{0}(B)^{+}$.

Note that for a finite topological space $X$, a locally closed subset $Y$ of $X$, and an open subset $U$ of $Y$, the maps $i_{U}^{Y}: \mathrm{K}_{0}(A(U)) \rightarrow \mathrm{K}_{0}(A(Y))$ and $r_{Y}^{Y \backslash U}: \mathrm{K}_{0}(A(Y)) \rightarrow$ $\mathrm{K}_{0}(A(Y \backslash U))$ are positive.

Definition 9.1. For $C^{*}$-algebras $A$ and $B$ over a finite topological space $X$, an $\mathcal{S} \mathcal{T}$ module homomorphism $\varphi: \mathrm{FK}_{\mathcal{S T}}(A) \rightarrow \mathrm{FK}_{\mathcal{S T}}(B)$ is called positive if the induced maps $\mathrm{FK}_{Y}^{0}(A) \rightarrow \mathrm{FK}_{Y}^{0}(B)$ are positive for all $Y \in \mathbb{L} \mathbb{C}(X)$, and an $\mathcal{S} \mathcal{T}$-module isomorphism $\mathrm{FK}_{\mathcal{S T}}(A) \rightarrow \mathrm{FK}_{\mathcal{S T}}(B)$ is called an order isomorphism if the induced isomomorphisms are order isomorphisms. For the reduced versions $\mathrm{FK}_{\mathcal{R}}, \mathrm{FK}_{\mathcal{B}}$, and $\mathrm{FK}_{\mathcal{B R}}$ of filtered K-theory, analogous definitions apply.

We are indebted to Mikael Rørdam for the elegant proof of the following lemma.

Lemma 9.2. Let $A$ be a real-rank-zero $C^{*}$-algebra and let $I$ and $J$ be (closed, twosided) ideals in $A$ satisfying $I+J=A$. Then any projection $p$ in $A$ can be written as $p=q+q^{\prime}$ with a projection $q$ in $I$ and a projection $q^{\prime}$ in $J$.

Proof. Let $p$ a projection in $A$ be given and write $p=a+b$ with $a \in I$ and $b \in J$. We may assume that $a=p a p$ and $b=p b p$. As $A$ has real rank zero, the hereditary subalgebra $p I p$ has an approximate unit of projections, so there exists a projection $q$ in $p I p$ satisfying $\|a-a q\|<1$. Since $q=p q p, q \leq p$ and we may define a projection $q^{\prime}$ as $q^{\prime}=p-q$. It remains to prove $q^{\prime} \in J$. We have

$$
\left\|q^{\prime}-q^{\prime} b q^{\prime}\right\|=\left\|q^{\prime}(p-b) q^{\prime}\right\|=\left\|q^{\prime} a(p-q)\right\| \leq\left\|q^{\prime}\right\|\|a-a q\|<1 .
$$

Since $q^{\prime} b q^{\prime} \in J$, the image of $q^{\prime}$ in the quotient $A / J$ is a projection of norm strictly less than 1 . Since such a projection is 0 , we get $q^{\prime} \in J$. 
The following theorem is a version of Corollary 5.19 taking the order into account.

Theorem 9.3. Let $X$ be an EBP space, and let $A$ and $B$ be $C^{*}$-algebras over $X$ of real rank zero. Then for any order isomorphism $\varphi: \operatorname{FK}_{\mathcal{B}}(A) \rightarrow \mathrm{FK}_{\mathcal{B}}(B)$ there is a unique order isomorphism $\Phi: \operatorname{FK}_{\mathcal{S} \mathcal{T}}(A) \rightarrow \mathrm{FK}_{\mathcal{S T}}(B)$ satisfying $\mathfrak{F}_{\mathcal{B}}(\Phi)=\varphi$.

Proof. By Corollary 5.19, $\Phi$ is an isomorphism if and only if $\varphi$ is. Assume that $\varphi$ is an order isomorphism, and let us show first for $Y \in \mathbb{O}(X)$ and then for $Y \in \mathbb{L} \mathbb{C}(X)$ that $\Phi_{Y}^{0}$ is an order isomorphism.

For $U$ an open subset of $X$, the following diagram has commuting squares and its rows are exact by Lemmas 4.7 and 4.8 .

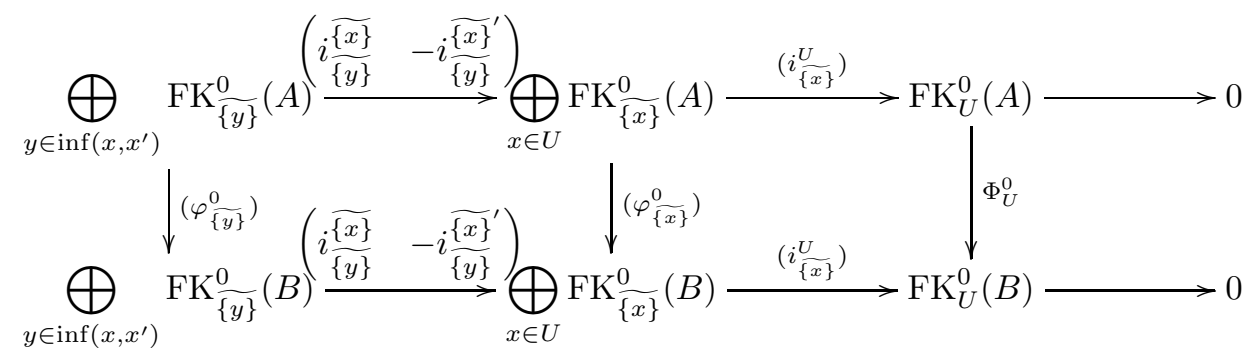

Since $(A(\widetilde{\{x\}}))_{x \in U}$ is a finite collection of ideals in $A(U)$, we see by Lemma 9.2 that the map $\left(i \frac{U}{\{x\}}\right): \bigoplus_{x \in U} \mathrm{~K}_{0}\left(A(\widetilde{\{x\}}) \rightarrow \mathrm{K}_{0}(A(U))\right.$ surjects $\bigoplus_{x \in U} \mathrm{~K}_{0}\left(A(\widetilde{\{x\}})^{+}\right.$onto $\mathrm{K}_{0}(A(U))^{+}$. Similarly, the map $\left(i \frac{U}{\{x\}}\right): \bigoplus_{x \in U} \mathrm{~K}_{0}\left(B(\widetilde{\{x\}}) \rightarrow \mathrm{K}_{0}(B(U))\right.$ surjects $\bigoplus_{x \in U} \mathrm{~K}_{0}\left(B(\widetilde{\{x\}})^{+}\right.$onto $\mathrm{K}_{0}(B(U))^{+}$. A simple diagram chase therefore shows that $\Phi_{U}^{0}$ is an order isomorphism since the map $\varphi_{\{x\}}^{0}$ is an order isomorphism for all $x \in U$.

For a locally closed subset $Y$ of $X$, choose open subsets $U$ and $V$ of $X$ satisfying $V \subseteq U$ and $U \backslash V=Y$. Then $\Phi_{U}^{0}$ is an order isomorphism. Consider the following diagram with exact rows and commuting squares.

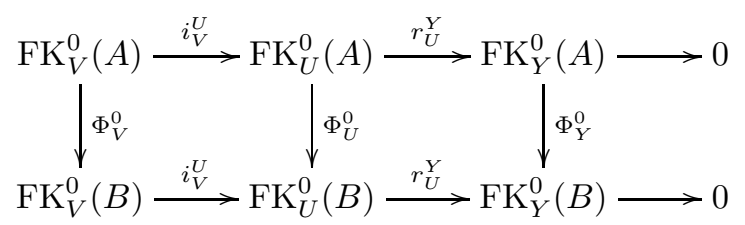

In [10, Theorem 3.14], Lawrence G. Brown and Gert K. Pedersen showed that given an extension $I \hookrightarrow C \rightarrow C / I$ of $C^{*}$-algebras, the $C^{*}$-algebra $C$ has real rank zero if and only if $I$ and $C / I$ have real rank zero and projections in $C / I$ lift to projections in $C$. Thus, since $A$ and therefore $M_{n} \otimes A(U)$ for all $n$ has real rank zero, the map $i_{U}^{Y}: \mathrm{K}_{0}(A(U)) \rightarrow \mathrm{K}_{0}(A(Y))$ surjects $\mathrm{K}_{0}(A(U))^{+}$onto $\mathrm{K}_{0}(A(Y))^{+}$. Similarly, the map $i_{U}^{Y}: \mathrm{K}_{0}(B(U)) \rightarrow \mathrm{K}_{0}(B(Y))$ surjects $\mathrm{K}_{0}(B(U))^{+}$onto $\mathrm{K}_{0}(B(Y))^{+}$. A simple diagram chase therefore shows that $\Phi_{Y}^{0}$ is an order isomorphism.

We have the following ordered analogs of Theorem 7.8 and Corollary 7.15 
Theorem 9.4. Let $X$ be a unique path space, and let $A$ and $B$ be $C^{*}$-algebras over $X$ of real rank zero. Assume that $\mathrm{K}_{1}(A(\{x\}))$ and $\mathrm{K}_{1}(B(\{x\}))$ are free abelian groups for all non-open points $x \in X$. Then for any order isomorphism $\varphi: \operatorname{FK}_{\mathcal{R}}(A) \rightarrow \mathrm{FK}_{\mathcal{R}}(B)$ there exists a (not necessarily unique) order isomorphism $\Phi: \operatorname{FK}_{\mathcal{B R}}(A) \rightarrow \operatorname{FK}_{\mathcal{B R}}(B)$ that satisfies $\mathfrak{F}_{\mathcal{B R}, \mathcal{R}}(\Phi)=\varphi$.

Proof. Since the functor $\mathfrak{F}_{\mathcal{B R}, \mathcal{R}}$ only forgets $\mathrm{K}_{1}$-groups, the desired follows immediately from Theorem 7.8 .

Corollary 9.5. Let $X$ be an EBP space, and let $A$ and $B$ be $C^{*}$-algebras over $X$ of real rank zero. Assume that $\mathrm{K}_{1}(A(\{x\}))$ and $\mathrm{K}_{1}(B(\{x\}))$ are free abelian groups for all $x \in X$. Then for any order isomorphism $\varphi: \operatorname{FK}_{\mathcal{R}}(A) \rightarrow \operatorname{FK}_{\mathcal{R}}(B)$ there exists a order isomorphism $\Phi: \mathrm{FK}_{\mathcal{S T}}(A) \rightarrow \mathrm{FK}_{\mathcal{S T}}(B)$ that satisfies $\mathfrak{F}_{\mathcal{R}}(\Phi)=\varphi$.

Proof. Combine the previous two theorems.

\section{Corollaries FOR ACCORDiON SPACES}

We summarize our results in the most satisfying case of accordion spaces. By combining Theorems 3.11, 5.17, 6.12 and Corollaries 6.9, 7.14 in the stable case and Proposition 8.5. Corollaries 6.9, 8.7, 8.8, and Theorem 8.10 in the unital case, we obtain the following characterization of purely infinite graph $C^{*}$-algebras, and of purely infinite Cuntz-Krieger algebras. In the first list, we use that the stabilization of a graph $C^{*}$-algebra is again a graph $C^{*}$-algebra by [1, Proposition 9.8(3)].

Corollary 10.1. Let $X$ be an accordion space. The different versions of filtered $\mathrm{K}$-theory introduced in this article induce bijections between the sets of isomorphism classes of objects in the following three lists, respectively.

List 1:

- tight, stable, purely infinite graph $C^{*}$-algebras over $X$,

- stable Kirchberg $X$-algebras A of real rank zero with all simple subquotients in the bootstrap class satisfying that $\mathrm{K}_{1}(A(\{x\}))$ is free for all $x \in X$,

- countable, exact, real-rank-zero-like $\mathcal{N} \mathcal{T}$-modules $M$ with $M(\{x\}, 1)$ free for all $x \in X$,

- countable, exact $\mathcal{B}$-modules $M$ with $M\left(x_{1}\right)$ free for all $x \in X$,

- countable, exact $\mathcal{R}$-modules $M$ with $M\left(\bar{x}_{1}\right)$ free for all $x \in X$.

\section{List 2:}

- tight, unital, purely infinite graph $C^{*}$-algebras over $X$,

- unital Kirchberg $X$-algebras $A$ of real rank zero, with all simple subquotients in the bootstrap class such that, for all $x \in X$, the group $\mathrm{K}_{1}(A(\{x\}))$ is free and

$$
\operatorname{rank} \mathrm{K}_{1}(A(\{x\})) \leq \operatorname{rank} \mathrm{K}_{0}(A(\{x\}))<\infty,
$$

- countable, exact, real-rank-zero-like pointed $\mathcal{N} \mathcal{T}$-modules $M$ such that, for all $x \in X$, the group $M(\{x\}, 1)$ is free and

$$
\operatorname{rank}(M(\{x\}, 1)) \leq \operatorname{rank}(M(\{x\}, 0))<\infty,
$$

- countable, exact pointed $\mathcal{B}$-modules $M$ such that, for all $x \in X$, the group $M\left(x_{1}\right)$ is free and

$$
\operatorname{rank}\left(M\left(x_{1}\right)\right) \leq \operatorname{rank}\left(\operatorname{coker}\left(\bigoplus_{y \rightarrow x} M\left(\widetilde{y}_{0}\right) \rightarrow M\left(\widetilde{x}_{0}\right)\right)\right)<\infty,
$$


- isomorphism classes of countable, exact pointed $\mathcal{R}$-modules $M$ such that, for all $x \in X$, the group $M\left(\bar{x}_{1}\right)$ is free and

$$
\operatorname{rank}\left(M\left(x_{1}\right)\right) \leq \operatorname{rank}\left(\operatorname{coker}\left(M\left(\widetilde{\partial} x_{0}\right) \rightarrow M\left(\widetilde{x}_{0}\right)\right)\right)<\infty .
$$

\section{List 3:}

- tight, purely infinite Cuntz-Krieger algebras over X,

- unital Kirchberg $X$-algebras $A$ of real rank zero, with all simple subquotients in the bootstrap class such that, for all $x \in X$, the group $\mathrm{K}_{1}(A(\{x\}))$ is free and

$$
\operatorname{rank} \mathrm{K}_{1}(A(\{x\}))=\operatorname{rank} \mathrm{K}_{0}(A(\{x\}))<\infty,
$$

- countable, exact, real-rank-zero-like pointed $\mathcal{N} \mathcal{T}$-modules $M$ such that, for all $x \in X$, the group $M(\{x\}, 1)$ is free and

$$
\operatorname{rank}(M(\{x\}, 1))=\operatorname{rank}(M(\{x\}, 0))<\infty,
$$

- countable, exact pointed $\mathcal{B}$-modules $M$ such that, for all $x \in X$, the group $M\left(x_{1}\right)$ is free and

$$
\operatorname{rank}\left(M\left(x_{1}\right)\right)=\operatorname{rank}\left(\operatorname{coker}\left(\bigoplus_{y \rightarrow x} M\left(\widetilde{y}_{0}\right) \rightarrow M\left(\widetilde{x}_{0}\right)\right)\right)<\infty,
$$

- countable, exact pointed $\mathcal{R}$-modules $M$ such that, for all $x \in X$, the group $M\left(\bar{x}_{1}\right)$ is free and

$$
\operatorname{rank}\left(M\left(x_{1}\right)\right)=\operatorname{rank}\left(\operatorname{coker}\left(M\left(\widetilde{\partial} x_{0}\right) \rightarrow M\left(\widetilde{x}_{0}\right)\right)\right)<\infty .
$$

\section{REFERENCES}

[1] Gene Abrams and Mark Tomforde, Isomorphism and Morita equivalence of graph algebras, Trans. Amer. Math. Soc. 363 (2011), 3733-3767.

[2] Sara E. Arklint, Do phantom Cuntz-Krieger algebras exist? (2012), available at arXiv: 1210.6515

[3] Sara Arklint, Rasmus Bentmann, and Takeshi Katsura, The K-theoretical range of CuntzKrieger algebras (2013), available at arXiv:1309.7162v1

[4] Sara Arklint, Gunnar Restorff, and Efren Ruiz, Filtrated K-theory of real rank zero $C^{*}$ algebras, Internat. J. Math. 23 (2012), no. 8, 1250078, 19, DOI 10.1142/S0129167X12500784.

[5] Sara E. Arklint and Efren Ruiz, Corners of Cuntz-Krieger algebras (2012), available at arXiv: 1209.4336

[6] Rasmus Bentmann, Filtrated K-theory and classification of $C^{*}$-algebras (University of Göttingen, 2010), available at www.math.ku.dk/ bentmann/thesis.pdf Diplom thesis.

[7] _ Kirchberg $X$-algebras with real rank zero and intermediate cancellation (2013), available at arXiv:math/1301.6652.

[8] Rasmus Bentmann and Manuel Köhler, Universal Coefficient Theorems for $C^{*}$-algebras over finite topological spaces (2011), available at arXiv:math/1101.5702v3.

[9] Mike Boyle and Danrun Huang, Poset block equivalence of integral matrices, Trans. Amer. Math. Soc. 355 (2003), no. 10, 3861-3886 (electronic), DOI 10.1090/S0002-9947-03-02947-7.

[10] Lawrence G. Brown and Gert K. Pedersen, $C^{*}$-algebras of real rank zero, J. Funct. Anal. 99 (1991), no. 1, 131-149, DOI 10.1016/0022-1236(91)90056-B.

[11] Joachim Cuntz, Simple $C^{*}$-algebras generated by isometries, Comm. Math. Phys. 57 (1977), no. $2,173-185$.

[12] Joachim Cuntz and Wolfgang Krieger, A class of $C^{*}$-algebras and topological Markov chains, Invent. Math. 56 (1980), no. 3, 251-268, DOI 10.1007/BF01390048.

[13] Søren Eilers, Gunnar Restorff, and Efren Ruiz, Classifying $C^{*}$-algebras with both finite and infinite subquotients (2010), available at arXiv:1009.4778

[14] _ Strong classification of extensions of classifiable $C^{*}$-algebras (2013), available at arXiv:1301.7695v1 
[15] Jeong Hee Hong and Wojciech Szymański, Purely infinite Cuntz-Krieger algebras of directed graphs, Bull. London Math. Soc. 35 (2003), no. 5, 689-696, DOI 10.1112/S0024609303002364.

[16] Eberhard Kirchberg, The classification of Purely Infinite $C^{*}$-algebras using Kasparov's Theorey. to appear in the Fields Institute Communication series.

[17] _ Das nicht-kommutative Michael-Auswahlprinzip und die Klassifikation nichteinfacher Algebren, $C^{*}$-algebras (Münster, 1999), 2000, pp. 92-141.

[18] Hua Xin Lin and Mikael Rørdam, Extensions of inductive limits of circle algebras, J. London Math. Soc. (2) 51 (1995), no. 3, 603-613, DOI 10.1112/jlms/51.3.603.

[19] Ralf Meyer and Ryszard Nest, $C^{*}$-algebras over topological spaces: filtrated K-theory, Canad. J. Math. 64 (2012), no. 2, 368-408, DOI 10.4153/CJM-2011-061-x.

[20] $\longrightarrow C^{*}$-algebras over topological spaces: the bootstrap class, Münster J. Math. 2 (2009), $215-252$.

[21] Cornel Pasnicu and Mikael Rørdam, Purely infinite $C^{*}$-algebras of real rank zero, J. Reine Angew. Math. 613 (2007), 51-73, DOI 10.1515/CRELLE.2007.091.

[22] N. Christopher Phillips, A classification theorem for nuclear purely infinite simple $C^{*}$ algebras, Doc. Math. 5 (2000), 49-114 (electronic).

[23] Gunnar Restorff, Classification of Cuntz-Krieger algebras up to stable isomorphism, J. Reine Angew. Math. 598 (2006), 185-210, DOI 10.1515/CRELLE.2006.074.

[24] Gunnar Restorff and Efren Ruiz, On Rørdam's classification of certain $C^{*}$-algebras with one non-trivial ideal. II, Math. Scand. 101 (2007), no. 2, 280-292.

[25] Mikael Rørdam, Classification of Cuntz-Krieger algebras, K-Theory 9 (1995), no. 1, 31-58, DOI 10.1007/BF00965458.

[26] Classification of extensions of certain $C^{*}$-algebras by their six term exact sequences in K-theory, Math. Ann. 308 (1997), no. 1, 93-117, DOI 10.1007/s002080050067.

[27] The Stacks Project Authors, Stacks Project. available online at: http://math.columbia.edu/algebraic_geometry/stacks-gi

[28] Andrew S. Toms and Wilhelm Winter, Strongly self-absorbing $C^{*}$-algebras, Trans. Amer. Math. Soc. 359 (2007), no. 8, 3999-4029, DOI 10.1090/S0002-9947-07-04173-6.

Department of Mathematical Sciences, University of Copenhagen, Universitetsparken 5, DK-2100 Copenhagen, Denmark

E-mail address: arklint@math.ku.dk

Department of Mathematical Sciences, University of Copenhagen, Universitetsparken 5, DK-2100 Copenhagen, Denmark

E-mail address: bentmann@math.ku.dk

Department of Mathematics, Keio University, 3-14-1 Hiyoshi, Kouhoku-ku, YokoHAMA 223-8522, JAPAN

E-mail address: katsura@math.keio.ac.jp 